Pure and Applied Mathematics Quarterly

Volume 4, Number 1

(Special Issue: In honor of

Gregory Margulis, Part 2 of 2$)$

$1-69,2008$

\title{
A Summary of the Work of Gregory Margulis
}

\author{
Lizhen Ji
}

\section{INTRODUCTION}

Gregory Margulis is a mathematician of great depth and originality. Besides his celebrated results on super-rigidity and arithmeticity of irreducible lattices of higher rank semisimple Lie groups, and the solution of the Oppenheim conjecture on values of irrational indefinite quadratic forms at integral points, he has also initiated many other directions of research and solved a variety of famous open problems. As Tits said in [Ti1, §5]:

"Margulis has completely or almost completely solved a number of important problems in the theory of discrete subgroups of Lie groups, problems whose roots lie deep in the past and whose relevance goes far beyond that theory itself. It is not exaggerated to say that, on several occasions, he has bewildered the experts by solving questions which appeared to be completely out of reach at the time. He managed that through his mastery of a great variety of techniques used with extraodinary resources of skill and ingenuity. The new and most powerful methods he has invented have already had other important applications besides those for which they were created and, considering their generality, I have no doubt that they will have many more in the future."

Indeed, in his solution to the Oppenheim conjecture, the approach of using dynamics on homogeneous manifolds to study number theoretic questions has had far-reaching and effective applications (see $\S 40$ ). This is another instance of his ability of combining Lie group theory and ergodic theory and applying them to seemly unrelated fields. ${ }^{1}$ As Howe pointed out in a survey titled "A century of Lie theory" [Ho, p. 262] more than 10 years after the above article of Tits:

\footnotetext{
Received September 14, 2007.

${ }^{1}$ On more than one occasions, Borel mentioned that Margulis was the first person who caused confusion between two Borels in the Borel measure and the Borel subgroups, by using both Lie theory (or rather algebraic group theory) and ergodic theory simultaneously. He also declared that he was not related to the other Borel.
} 
"The influence between Lie theory and ergodic theory has been mutual. A particularly striking example of this was Margulis's use of ergodic theory in the proof of his Superrigity Theorem [Zi1], which was then reinterpreted as being a result in ergodic theory by Zimmer. A very recent example of this mutual interaction is the Margulis proof of the Oppenheim Conjecture, followed quickly by Ratner's broad generalization of the key ergodic-theoretic result underlying his proof [Ra1-6]."

The impact of this important work of Margulis on the Oppenheim conjecture was also confirmed by Borel [Bo3, p. 14]:

"G.A.Margulis ... proved the Oppenheim conjecture... This breakthrough opened the way to an intense activity both on refinements of the Oppenheim conjecture, on the Raghunathan conjecture and a related one by Dani."

It is also well-known that the Margulis lemma is a basic tool in hyperbolic geometry and more generally geometry of manifolds of nonpositive curvature and convergence and collapsing of manifolds (see $\S 6$ ), and his result on the generalized prime number theorem on lengths of closed geodesics has generated an active theory covering several different fields (see §14). His explicit construction of expander graphs and the solution of a long-standing problem of Banach-Ruziewicz on the uniqueness of finitely additive, rotation invariant measure on the $n$-sphere $S^{n}$ are two other results of great originality (see $\S 16$ and $\S 17$ ).

In order to convey a sense of the width and depth of his work, this article tries to summarize some major results of Margulis. For a very good summary of some earlier results of Margulis, especially his work on arithmeticity and super-rigidity of lattices of higher rank semisimple Lie groups, see the article [Ti1] by Tits, written for the occasion when Margulis was awarded a Fields medal in 1978 in Helsinki.

The current article is divided into topics which are arranged roughly according to the times they were first considered by Margulis or according to the years of publication in MathSciNet. ${ }^{2} 3$

Descriptions are usually brief for many obvious reasons, for example, due to lack of knowledge of the author about many topics covered by the work of Margulis. But, whenever possible and appropriate, we will try to explain a bit of

\footnotetext{
${ }^{2}$ Another method to organize this artcle, which might be better, is to group papers according to the topics under discussion. We have tried to do this to certain extent by putting several papers into one section under a common title, for example, the sections on arithmeticity and super-rigidity of higher rank lattices summarize many papers of Margulis. But the arrangement according to the publication dates in this article might give a historic sense to the work of Margulis. To help organize this paper and orient the reader, we have inserted a detailed table of contents.

${ }^{3}$ The paper [67] is not listed in MathSciNet for some reason.
} 
history of the results under discussion, contributions of Margulis, and some later developments. ${ }^{4}$

The references near the end of this article are divided into two parts: the first part consists of papers and books by Margulis which are indexed by the years in which they were published, and the second part consists of some publications by others cited in this article, which are not meant to be comprehensive, and hence some important references might not be cited.

Acknowledgments. I would like to thank G.Margulis for very valuable and informative email correspondences about his early mathematical life and developments of some of his mathematical contributions, A.Lubotzky for helpful references and careful comments on an earlier version, G.Prasad for helpful conversations and advice, A.Reid, Y.Segev for helpful references, R.Spatzier for inspiring conversations and comments, D.Kleinbock for carefully reading earlier versions and many helpful suggestions, comments and references, H.Oh for many helpful references and comments on earlier versions, and D.Fisher for many helpful comments and references. I would also like to thank M.Ratner, M.Burger and A.Eskin for helpful comments and references. The author was partially supported by NSF grant DMS 0604878 during the preparation of this article.

\section{Contents}

1. Introduction 1

2. Results proved during the undergraduate years 5

3. Positive harmonic functions on nilpotent groups 6

4. Exponential growth of the fundamental group and Anosov flow 6

5. Volume of locally symmetric spaces and existence of unipotent elements

6. Margulis Lemma 9

7. Arithmeticity of lattices 10

8. Local and strong rigidity of lattices and locally symmetric spaces 14

9. Super-rigidity of lattices 17

10. Normal subgroups of lattices 19

\footnotetext{
${ }^{4}$ It should be emphasized right from the beginning that there have been many significant results in various directions after the original work of Margulis. It seems difficult to mention all of them in this article. Though some experts have been consulted about such results and papers, all the responsibility rests with the author. I would like to apologize in advance to those whose important works are not described or mentioned below.
} 
11. Normal subgroups of groups of rational points and Margulis-Platonov conjectures

12. Characterization of arithmetic groups

13. Further developments on rigidity of lattices

14. Counting lengths of geodesics and volume entropy, and Margulis measure

15. Long time behavior of unipotent group orbits

16. Expander graphs and work in combinatorics

17. Invariant measures on spheres and $\mathbb{R}^{n}$

18. Strong approximation of algebraic groups

19. Maximal subgroups of lattices and linear groups

20. Decomposition of lattice subgroups into amalgams

21. Actions of affine transformations, Milnor conjecture, Auslander conjecture

22. Absence of invariant analytic hypersurfaces.

23. Proportionality of covolume of lattices

24. The Oppenheim conjecture on values of quadratic forms

25. Quantitative version of the Oppenheim conjecture

26. Oseledets multiplicative ergodic theorem and Lyapunov exponents

27. Proximal linear maps

28. QC-maps and Carnot-Carathéodory spaces

29. Bounded orbits and unbounded orbits of flows

30. Compact quotients of homogeneous spaces

31. Metric Diophantine approximation and Khintchine-type theorems 46

32. Hyperbolic Penrose tile 47

33. Wiener ergodicity for semisimple Lie groups

34. Finite coverings of hyperbolic manifolds 49

35. A generalized Tits alternative 50

36. Rigidity of actions of Lie groups and lattices 50

37. Random walks on homogeneous spaces and reduction theory

38. Coarse metrics on reductive groups 
39. Effective ergodic theory 54

40. Problem lists by Margulis $\quad 55$

References to the publication of Margulis $\quad 58$

References to papers by others $\quad 62$

\section{Results PROVED DURING THE UNDERGRADUATE YEARS}

Margulis wrote three papers [66] [67] [68] when he was an undergraduate student at the Moscow State University. Even though it was not unusual for undergraduate students of Moscow State University to do research, what he has achieved was very significant. In fact, the paper [68] was reported by Borel [Bo2] at the famed Bourbaki seminar.

He wrote his first paper [66] when he was a third year undergraduate student and attended Dynkin's seminar. At that time, structure of positive harmonic functions was one of the problems studied by Dynkin.

Starting at the end of the third year, Sinai became his advisor. Margulis' second paper [67] proved exponential growth of the fundamental group of a 3dimensional manifold admitting Anosov flows, and it was published as an appendix to a survey by Anosov and Sinai [AS]. Participation in Sinai's seminar and discussions with him and his other students gave Margulis valuable experience in ergodic theory and dynamical systems, and it played a very important role in his mathematical career. In fact, his thesis is titled On some aspects of the theory of Anosov flows [04-1], which includes the counting of closed geodesics and lattice points in terms of the entropy and a construction of an important measure, now called the Margulis measure (or Bowen-Margulis measure) (see §14). Ergodic theory and dynamical systems are crucial in his celebrated later works such as arithmeticity and super-rigidity of irreducible lattices in higher rank semisimple Lie groups and the solution of the Oppenheim conjecture etc.

At the end of his undergraduate study, Margulis wrote a well-known paper [68] with his classmate D.Kazhdan, which solved several outstanding problems, including a conjecture of Selberg on the existence of unipotent elements in nonuniform lattices. The well-known Margulis Lemma was also motivated by a result proved in this paper (see $\S 6$ ).

After this paper [68], Margulis became very much involved in the theory of discrete subgroups of Lie groups. In particular he started to work on the problem of arithmeticity of nonuniform lattices. He succeeded after several years by using the approach based on the study of unipotent elements in a nonuniform lattice and on a nondivergence result for actions of unipotent groups on the space of lattices. 
It should be noted that the general strategy associated with this approach had been introduced earlier by Piatetski-Shapiro and Selberg (see $\S 7$ and $\S 9$ ).

\section{Positive harmonic FunCtions on nilpotent Groups}

Harmonic functions on $\mathbb{R}^{n}$ are classical objects and have been studied from various points of view. A famous result of Liouville says that every positive harmonic function on $\mathbb{R}^{n}$ is constant. In particular, every bounded harmonic function on $\mathbb{R}^{n}$ is constant too. (Note that constant functions are harmonic.) The former corresponds to the fact that the Martin boundary of $\mathbb{R}^{n}$ is trivial, and the latter corresponds to the fact that the Poisson boundary of $\mathbb{R}^{n}$ is trivial.

There are several natural ways to generalize these results. One important problem is to consider harmonic functions on other homogeneous spaces, for example, on nilpotent Lie groups, solvable Lie groups and symmetric spaces. Since $\mathbb{R}^{n}$ is an abelian Lie group, a particularly natural class of groups consists of nilpotent Lie groups.

Another problem is to consider discrete versions of these homogeneous spaces, for example, uniform lattices acting on these spaces. In this sense, $\mathbb{Z}^{n}$ corresponds to $\mathbb{R}^{n}$. The notion of harmonic functions on a finitely generated group $\Gamma$ can be defined in terms of a non-negative measure $p$ on $\Gamma$ whose support contains a set of generators. Briefly, a function $f$ is harmonic if the mean value equality holds, i.e., for every point $x \in \Gamma$, the following equality is true:

$$
f(x)=\sum_{\gamma \in \Gamma} p(\gamma) f(\gamma x) .
$$

If $\Gamma$ is nilpotent and $p$ is a probability measure, then every bounded harmonic function is constant. See $[\mathrm{Dy}]$ for a survey on these results.

In [66], it is shown how to reduce a positive harmonic function $f$ on a nilpotent group $\Gamma$ to its abelian quotient $\Gamma /[\Gamma, \Gamma]$. Briefly, such a function $f$ is constant on every coset of $[\Gamma, \Gamma]$. Hence, $[66]$ is related to the Martin boundary of nilpotent groups.

Determination of the Poisson boundary and the Martin boundary of nilpotent and solvable groups are still actively pursued. See $[\mathrm{DaH}][\mathrm{Bab}]$ and the references there.

\section{Exponential growth of the fundamental group and Anosov flow}

Given a finitely generated group $\Gamma$, we can define the notion of growth rates. Briefly, for any finite set of generators $S$ of $\Gamma$, there is an associated word metric $d_{S}$ on $\Gamma$, which can be taken to be left invariant. Then the group $\Gamma$ is said to be 
of exponential growth if the number of elements of $\Gamma$ in a metric ball of radius $R$ with respect to $d_{S}$ grows exponentially in $R$.

Though the word metric $d_{S}$ and hence the exact sizes of metric balls depend on the choice of the generating set $S$, the notion of exponential growth of $\Gamma$ does not depend on the choice, and the exponential growth rate is an important large scale invariant of the group $\Gamma$.

The main result of the paper [67] says that if a three dimensional manifold $M$ admits an Anosov flow, then the fundamental group of $M$ has exponential growth.

This puts an algebraic topological restriction on three dimensional manifolds which admit Anosov flows.

It might be helpful to understand this result in the following context. It was known (see $[\mathrm{AS}]$ ) that if $M$ is a compact Riemannian manifold with strictly negative sectional curvature, then the geodesic flow of $M$ is an Anosov flow.

On the other hand, shortly after the paper [67], Milnor [Mi] proved a wellknown result that if $M$ is a compact Riemannian manifold with strictly negative sectional curvature, then the fundamental group of $M$ has exponential growth.

\section{Volume of LOCALly Symmetric SPACES AND EXISTENCE OF UNIPOTENT ELEMENTS}

If the volume of a Riemannian manifold (or orbifold) is finite, then it provides an important basic geometric invariant of the manifold. It is a multiplicative in the following sense. Let $X$ is a Riemannian manifold, and $\Gamma_{1}, \Gamma_{2}$ two discrete groups acting isometrically on $X$ with finite volume quotients $\Gamma_{1} \backslash X$ and $\Gamma_{2} \backslash X$. Assume that $\Gamma_{1} \subset \Gamma_{2}$. Then

$$
\operatorname{vol}\left(\Gamma_{1} \backslash X\right) / \operatorname{vol}\left(\Gamma_{2} \backslash X\right)=\left[\Gamma_{2}: \Gamma_{1}\right] .
$$

This implies that if $\Gamma_{1}$ is small, the volume $\operatorname{vol}\left(\Gamma_{1} \backslash X\right)$ is big.

If $X$ is a Riemannian symmetric space of noncompact type and $\Gamma$ is a discrete isometry group with $\operatorname{vol}(\Gamma \backslash X)<+\infty$, i.e., $\Gamma$ is a lattice, then in many cases, it is easy to construct subgroups $\Gamma^{\prime}$ with arbitrary large index $\left[\Gamma: \Gamma^{\prime}\right]$. In other words, there is no finite upper bound on volumes of locally symmetric spaces $\Gamma \backslash X$ modeled on each given symmetric space $X$.

The first striking result in the joint paper of Margulis with Kazhdan [68] is that there is a uniform positive lower bound for the volumes of all quotients $\Gamma \backslash X$, which only depends on the invariant Riemannian metric on $X$.

When $X$ is equal to the Poincare upper half plane $\mathbb{H}^{2}=\{x+i y \mid x \in \mathbb{R}, y>0\}$, this result was known before and is classical (see [Si] [Bea, Chap. 10]). 
This result on the lower bound is a consequence of the following result: Let $G$ be a connected linear semisimple Lie group without compact factor. Let $\rho($,$) be$ a right invariant metric on $G$. There exist a neighborhood $\tilde{V}_{1}$ of e, and constants $C_{1}>0$ and $c>1$ such that for any discrete subgroup $\Gamma$ of $G$, one can find some $g \in G$ satisfying the two following conditions:

(1) $\rho(e, g) \leq C_{1}$,

(2) for any $\gamma \in \Gamma \cap \tilde{V}_{1}, \gamma \neq e$, the following inequality holds:

$$
\rho\left(e, g \gamma g^{-1}\right) \geq c \rho(e, \gamma) .
$$

The proof of this result is based on the following result: For every connected Lie group $G$, there exists a neighborhood $U$ of the identity element such that for every discrete subgroup $\Gamma$ in $G$, the elements in the intersection $\Gamma \cap U$ generate a nilpotent subgroup.

This latter result was the starting point of the Margulis lemma (see §6) and also used to prove the second major result of [68]. In fact, it was used by Kazhdan and Margulis [68] to prove a conjecture of Selberg [Se2, p. 180]: If $G$ is a connected linear semisimple Lie group, and $\Gamma \subset G$ is a non-uniform lattice, i.e., $\Gamma \backslash G$ has finite measure with respect to any Haar measure of $G$ but is not compact, then $\Gamma$ contains a nontrivial unipotent element.

When $G=S L(2, \mathbb{R})$, this conjecture was known earlier and unipotent elements correspond (or fix) the cuspidal (or infinite, or ideal) points of a Dirichlet fundamental domain for the $\Gamma$-action on $\mathbb{H}^{2}$. This conjecture is also known for arithmetic subgroups; in fact, it was the content of a conjecture of Godement for arithmetic subgroups, proved independently by Borel and Harish-Chandra $[\mathrm{BoHC}]$, and Mostow and Tamagawa [MoT]. (Non-uniform arithmetic subgroups contain many unipotent elements. Their intersection with $\mathbb{Q}$-parabolic subgroups contains lattices of the real locus of the unipotent radical of the parabolic subgroups.)

Among all discrete groups $\Gamma$ of $G$, lattices are particularly important for various reasons. First, arithmetic subgroups are lattices. Second, for the study of automorphic functions, this assumption is often needed (see [Se1, p. 101]).

The presence of unipotent elements in non-uniform lattices has played an important role in the study of rigidity and arithmeticity of $\Gamma$. See [Se1, §7.4] for one of the original motivations. Another motivation is that to study the Selberg trace formula, a precise description of the cusps (i.e., noncompact parts) of a good fundamental domain, for example a Dirichlet fundamental domain, is important [Se2]. If $\Gamma$ is either an arithmetic subgroup or a lattice acting on $\mathbb{H}^{2}$, the cusps are described by unipotent elements in $\Gamma$, and the above conjecture is the first step towards such goals. As Borel explained in [Bo3, p. 8], "This latter statement [on existence of unipotent elements] was also not unexpected, but it was 
an enormous surprise that both could be established so directly, using only basic facts about semisimple Lie groups (but in an amazingly ingenious way."

One consequence of the above uniform lower bound on $\operatorname{vol}(\Gamma \backslash X)$ is that if $X=G / K$ and $G$ does not contain any nontrivial compact factor, then every lattice $\Gamma \subset G$ is contained in some maximal lattice $\tilde{\Gamma}$, where by a maximal lattice, it means that $\tilde{\Gamma}$ is not contained properly in another lattice.

There have been a lot of work on computing the volume spectrum of $\Gamma \backslash X$ when $\Gamma$ changes, in particular, the best lower bound on the volumes. Another question concerns volumes of $\Gamma \backslash X$ when $\Gamma$ are arithmetic subgroups. To show differences of these two questions, one notes that when $X=\mathbb{H}^{2}$, or $\mathbb{H}^{3}$, the real hyperbolic space of dimension 2 or 3 , for every $v$, there are only finitely many arithmetic locally symmetric spaces $\Gamma \backslash X$ with $\operatorname{vol}(\Gamma \backslash X) \leq v$. On the other hand, there are infinitely many $\Gamma \backslash X$ with $\operatorname{vol}(\Gamma \backslash X) \leq v$ when $v \gg 0$. For some references on these topics, see $\S 23$ below (see also [Ji1] for more references).

\section{Margulis Lemma}

Another very important result, the so-called Margulis Lemma, was motivated by a result in [68]. It have played a fundamental role in the study of manifolds with non-positive curvature, in particular hyperbolic manifolds, discrete group actions on them, and convergence of Riemannian manifolds.

The basic motivation is as follows. Recall that on a complete Riemannian manifold $M$, the injectivity radius at a point $x$ is the maximum radius of the ball in the tangent space $T_{x} M$ with center at the origin which is mapped diffeomorphically into $M$ under the exponential map $\exp _{x}$. If $M$ is compact, there is a uniform lower bound for the injectivity radius for all points. This is not true in general for a noncompact Riemannian manifold $M$. But for many applications, it is important to understand local structures of manifolds on uniform sizes. For this purpose, it is crucial to understand points of $M$ where the injectivity radius is small. Another reason is that when we consider an infinite family of Riemannian manifolds $M_{j}$, to understand the convergence or collapsing properties of this family, we need also to understand local structures of $M_{j}$ on uniform sizes.

As stated in the previous section, one important result in [68] is as follows: For every connected Lie group $G$, there exists a neighborhood $U$ of the identity element such that for every discrete subgroup $\Gamma \subset G$, the elements in $\Gamma \cap U$ generate a nilpotent subgroup. The important point here is that the neighborhood $U$ is independent of the discrete subgroup $\Gamma .{ }^{5}$

In an unwritten work (or rather a private communication to Gromov) (see [Gr, p. 232]), Margulis proved the following result: For every $n \in \mathbb{N}$, there is a

\footnotetext{
${ }^{5}$ It was later realized that this result was actually proved by Zassenhaus in 1938.
} 
constant $\varepsilon>0$ depending only on $n$ such that if $V^{n}$ is a compact Riemannian manifold with sectional curvature strictly bounded between 0 and -1 , and two elements $\alpha, \beta \in \pi_{1}\left(V, v_{0}\right)$ can be represented by loops of length less than or equal to $\varepsilon$, then there is a natural number $m$ such that $\alpha^{m}$ and $\beta^{m}$ generate a nilpotent subgroup of $\pi_{1}\left(V, v_{0}\right)$.

In [Gr1], Gromov called this result Margulis Lemma. There are also various other versions of Margulis Lemma based on similar ideas. For applications to group actions on Riemannian manifolds, one version of the Margulis Lemma can be stated as follows [BaGS, p. 101, p. 107]: Let $X$ be a simply connected and nonpositively curved complete Riemannian manifold with sectional curvarure bounded from below by -1 and $\Gamma$ is a discrete group acting isometrically and properly on $X$. For any $\mu>0$ and $x \in X$, define a subgroup

$$
\Gamma_{\mu, x}=\langle\{\gamma \in \Gamma \mid d(x, \gamma x) \leq \mu\}\rangle .
$$

Then there exist a constant $\mu_{0}$ and an integer $I_{0}$ only depending on $n$ such that for every $\mu \leq \mu_{0}$ and $x \in X$, the subgroup $\Gamma_{\mu, x}$ is virtually nilpotent; in fact, it contains a nilpotent subgroup of index bounded uniformly by $I_{0}$.

As a consequence, if the sectional curvature of $X$ is strictly negative, then the thin part $(\Gamma \backslash X)_{\leq \mu}$ of such a quotient $\Gamma \backslash X$ is basically either a cusp or a neck, when the $\mu$-thin part is by definition the set of points $\bar{x} \in \Gamma \backslash X$ where the injectivity radius is less than $\mu$. The reason is that for every point $x \in X$, the injectivity radius of the image $\bar{x}$ of $x$ in $\Gamma \backslash X$ is equal to $\inf _{\gamma \in \Gamma} d(x, \gamma x)$, and its small neighborhoods are described by quotients of nilpotent subgroups (see [Fu, Theorem 4.6, p. 206]).

As mentioned earlier, another type of applications of the Margulis Lemma concern convergence and collapsing of families of Riemannian manifolds. They were initiated by Gromov and carried out extensively by Cheeger, Fukaya and Gromov et al. Basically, the presence of nilpotent structure is related to collapsing of Riemannian manifolds to lower dimensional ones. ${ }^{6}$ See $[\mathrm{Fu}]$ for a survey on generalizations of the Margulis Lemma in geometry and applications on convergence of Riemannian manifolds under bounds on curvatures and diameters, or other geometric conditions.

\section{Arithmeticity of lattices}

An important consequence of the uniformization theorem for Riemann surfaces is that there are in general positive dimensional families of non-conjugate lattices in $S L(2, \mathbb{R})$ (or rather $P S L(2, \mathbb{R})$ ). For example, if $\Gamma \subset S L(2, \mathbb{R})$ is a lattice

\footnotetext{
${ }^{6}$ This is closely related to the horospherical decomposition of symmetric spaces with respect to parabolic subgroups, and the collapsing of the Borel-Serre compactification of arithmetic locally symmetric spaces to the reductive Borel-Serre compactification.
} 
such that $\Gamma \backslash \mathbb{H}^{2}$ is a closed surface of genus $g \geq 2$, then $\Gamma$ belongs to a $(6 g-6)$ dimensional family of non-conjugate lattices, or equivalently their corresponding hyperbolic surfaces are not isometric.

This deformation, or equivalently non-rigidity, of such lattices implies immediately that most of lattices in $S L(2, \mathbb{R})$ are not arithmetic subgroups, since there are only countably many arithmetic subgroups.

On the other hand, for other semisimple linear Lie groups, it is difficult to construct non-arithmetic lattices. One reason is that there is no uniformization theorem for higher dimensional manifolds which we can use as above. (To see lack of any direct generalization of the uniformization theorem in one complex variable, note that all simply connected domains in $\mathbb{C}$ are biholomorphic to each other. But this is completely false for $\mathbb{C}^{n}, n \geq 2$; in fact, there are uncountably infinitely many of non-biholomorphic simply connected (or even contractible) domains in $\mathbb{C}^{n}$.)

After success with several special cases, Selberg made the following conjectures [Se1, p. 119] [Se3, §5]:

(1) Let $G$ be a linear semisimple Lie group, and $\Gamma \subset G$ an irreducible lattice. Then $\Gamma$ can be deformed into a group whose matrix representation has entries from some number field, and the denominators of these entries are uniformly bounded.

(2) If the real rank of $G$ is at least 2 and $\Gamma$ is a non-uniform lattice, then $\Gamma$ is an arithmetic subgroup of $G$ with respect to a suitable $\mathbb{Q}$-structure on $G$.

The first conjecture implies that if a lattice $\Gamma$ is (locally) rigid, i.e., does not admit nontrivial deformations, then $\Gamma$ has a matrix realization with entries given by algebraic numbers [Se3, p. 159, p. 164].

Earlier, the assumption that the rank of $G$ being at least 2 was not made for Conjecture (2), but only the group $S L(2, \mathbb{R})$ and its lattices were excluded. After some non-arithmetic lattices acting on the real hyperbolic spaces $\mathbb{H}^{n}, n=3,4$, were discovered, the conjecture of Selberg was modified (see [PS1, p.3]).

The importance of these conjectures, in particular the second one, is that the reduction theory for arithmetic subgroups can be used to understand the structure of neighborhoods at infinity of $\Gamma \backslash G$ (or $\Gamma \backslash X$ ), which is fundamental to the theory of automorphic forms for $\Gamma$. Indeed, as Selberg pointed out in [Se2, p. 180]:

"The most serious obstacle to carrying out of the idea sketched above is that, with the exception of the case of a hyperbolic plane (and of course for the product of a hyperbolic plane and euclidean or compact symmetric spaces) it is not known 
what conditions of the noncompactness but finite measure or volume of D imply about $\Gamma$."

He went on to say:

"The more general approach would be to make definite assumptions about the nature of the cusps and the subgroups of $\Gamma$ that describe them, namely that these be of the same nature as those encountered for the arithmetic subgroups of algebraic groups in the sense of Borel and Harish-Chandra. There are reasons to believe that perhaps all groups $\Gamma$ with finite volume of $D$ would fall in this category."

In fact, in the famous book of Langlands [Lan] (see also [OW]), several assumptions are made on lattices $\Gamma$ of semisimple Lie groups $G$ and their fundamental domains $D$ in the symmetric spaces $X=G / K$ right from the beginning. These assumptions are based on the reduction theory for arithmetic groups. Understanding structures of fundamental domains is also important for rigidity properties of locally symmetric spaces $\Gamma \backslash X$ (see [Se1, pp. 114-116]).

The first papers of Margulis towards the above conjecture of Selberg, i.e., arithmeticity of lattices, are in [69-1] [69-3]. One result states that if $G$ is a semisimple linear algebraic group defined over $\mathbb{R}$, has no compact factors and has $\mathbb{R}$-rank at least two, and $\Gamma \subset G$ is a non-uniform irreducible lattice, then $G$ admits a $\mathbb{Q}$-structure so that the subgroup $\Gamma \cap G_{\mathbb{Q}}$ is of finite index in $\Gamma$, and there exists a $\mathbb{Q}$-parabolic subgroup $P \subset G$ so that $\Gamma \cap P$ is an arithmetic subgroup of $P_{\mathbb{Q}}$.

In his review of the paper [69-3], Garland wrote:

"This theorem represents a substantial contribution to a problem raised by A.Selberg....The fundamental tools for the proof of the main theorem are (i) the methods and results of an earlier paper of D.A.Kazhdan and the author [68], and (ii) a result concerning the group $S L(n, \mathbb{Z})$, which we decribe presently. ... Now we describe the result concerning $S L(n, \mathbb{Z})$. Let $\{u(t)\}$ be a one-parameter unipotent subgroup of $S L(n, \mathbb{R})$ and let $p$ be the natural projection from $S L(n, \mathbb{R})$ to $X=S L(n, \mathbb{R}) / S L(n, \mathbb{Z})$; then there exists a compact subset $\Omega$ of $X$ so that for every $t_{0}>0$, there exists a $t>t_{0}$ so that $p(u(t))$ belongs to $\Omega$. Using (i) and (ii) the author then proves the existence of "amply many" unipotent in $\Gamma$. In particular, he proves that for a suitable parabolic subgroup $P$ in $G$, with unipotent radical $U$, one has that the quotient of $U$ by the intersection of $\Gamma$ and $U$ is compact. This is a key step in proving the theorem."

According to Margulis, this result (ii) quoted in the above paragraph is one of his best results. Indeed, as Tits wrote in [Ti1, p. 59], "the ... result ... was soon realized by the experts as a crucial step for the proof of Selberg conjecture." This result was stated as a theorem by Piatetski-Shapiro in [PS1], also in connection with the arithmeticity of nonuniform lattices, but he never gave a proof. The 
proof of (ii) is based on the phenomenon of polynomial divergence (applied to actions of $\{u(t)\}$ on the exterior products of $\mathbb{R}^{n}$ ) and on a rather sophisticated induction argument related to the geometry of numbers. The technique used in the proof of (ii) was refined and quantified first by S.G.Dani in [Da1] (in the proof of the quantitative recurrence of unipotent orbits to compact sets) and later in a joint work of Margulis with Kleinbock [98-2] and by Eskin, Mozes and Shah in their work on counting of integral points on homogeneous varieties [EMS].

Margulis' work on the above result (ii) ([71] or [75-3]) provided him with some intuition which played an important role in his much later work on unipotent flows (this intuition was based on the understanding of the importance of the polynomial divergence in the theory of unipotent flows).

The detailed proof of the main result announced in [69-3] was given in [74-2] and [75-2] (actually the paper [75-2] was submitted for publication much earlier than [74-2]). Another important ingredient in the proof is a construction from representation theory. This construction was used later by $\mathrm{Oh}[\mathrm{Oh} 4]$ in her work on discrete groups generated by lattices in horospherical subgroups. It should be mentioned that Raghunathan obtained an independent proof of the main result announced in [69-3]. ${ }^{7}$

In [75-2] Margulis also proved the strong rigidity for non-uniform lattices in higher rank groups using a totally different method (see the next section for more detail).

Reduction of the proof of arithmeticity of non-uniform lattices in higher rank groups to the main result announced in [69-3] was started in [74-2] and completed in $[75-1]$.

In [PS1, p. 3] (see also [PS2, p. 189]), Piatetski-Shapiro conjectured that irreducible uniform lattices $\Gamma$ in $G$ of rank at least 2 are also arithmetic. This is a big step from the conjecture of Selberg, since unipotent elements of non-uniform lattices $\Gamma$ play crucial roles in the proof of arithmeticity of $\Gamma$ in the above papers. He defined arithmetic subgroups of Lie groups which are not linear Lie groups. Briefly, a lattice $\Gamma$ in a semisimple Lie group $G$ is called arithmetic if there is a linear semisimple Lie group $\mathbf{G}^{\prime}$ defined over $\mathbb{Q}$ and a surjective homomorphism $\varphi: \mathbf{G}^{\prime}(\mathbb{R}) \rightarrow G$ with a compact kernel and an arithmetic subgroup $\Gamma^{\prime}$ of $\mathbf{G}^{\prime}(\mathbb{Q})$ such that $\varphi\left(\Gamma^{\prime}\right)$ is commensurable with $\Gamma$.

In [PS1, pp. 4-5], Piatetski-Shapiro also introduced arithmetic subgroups of linear p-adic Lie groups and products of both real Lie groups and linear p-adic Lie groups, and formulated questions on rigidity and arithmeticity of lattices in such locally compact groups.

\footnotetext{
${ }^{7}$ See the article $[$ Bo3, pp. $7-10, \S 10]$ for a very good summary of Raghunathan's contributions to strong rigidity and arithmeticity of lattices and a history of related results.
} 
The paper [74-1] [75-4] outlined a proof of this conjecture of arithmeticity of Piatetski-Shapiro on uniform irreducible lattices of semisimple Lie groups $G$ when the rank of the symmetric space associated with $G$ is at least two. The full details appeared in [77-2] [84-1].

A comprehensive discussion of these and other results is in the book [91-1]. Another account together with some generalizations on rigidity of group actions is given in the book [Zi1]. For a vivid description of the proof of arithmeticity theorem, see [Ti1] and also [Bo3, §7, pp. 7-10].

Besides non-arithmetic lattices acting on the real hyperbolic spaces [GPS], there are also non-arithmetic lattices acting on the complex hyperbolic spaces of low dimensions $[\mathrm{DeM}]$. On the other hand, lattices acting on the two types of other rank one symmetric spaces of noncompact type are arithmetic [Co] [GP]. Non-arithmetic lattices in rank $1 p$-adic semi-simple Lie groups always exist [Lu1].

In the above discussions, we concentrated on lattices in semisimple Lie groups. In fact, Margulis proved that if $S$ is a finite set of primes and $G_{p}$ is a semisimple $p$ adic Lie group with trivial center, any irreducible lattice in the product $\prod_{p \in S} G_{p}$ is arithmetic if the sum of the ranks of $G_{p}$ over $p \in S$ is at least 2 (see [91-1]).

In his lecture at Yale in 1992, Selberg suggested that some of his work should imply that any discrete subgroup containing lattices from the opposite horospherical subgroups of a product of $S L(2, \mathbb{R})$ is an arithmetic subgroup (or close to it). This is stronger than the arithmeticity of such discrete subgroups, since they may not be a priori lattices. Margulis raised this question for discrete subgroups $\Gamma$ of general higher rank semisimple Lie groups $G$ such that $\Gamma$ contain lattices from opposite horospherical subgroups and intersect any normal subgroups trivially, i.e., asked whether such groups $\Gamma$ are arithmetic under the above assumption. If $G$ is a split higher rank simple Lie group and not equal to $S L(3, \mathbb{R})$, then this propblem of Selberg and Margulis was solved positively by Oh [Oh4]. As mentioned above, a construction from representation theory by Margulis in [74-2] and [75-2] was used crucially here in [Oh4].

\section{LOCAL AND STRONG RIGIDITY OF LATTICES AND LOCALLY SYMMETRIC SPACES}

Arithmeticity of lattices is closely related to rigidity of lattices. In fact, one of the main motivations for Selberg is to use deformation rigidity to prove arithmeticity of lattices. On the other hand, rigidity of lattices is also natural from the point of view of geometry of locally symmetric spaces.

Besides [Se2] [Se3], the first papers on rigidity of lattices and locally symmetric spaces include $[\mathrm{Ca}][\mathrm{CaV}][\mathrm{We}]$. The motivation of $[\mathrm{CaV}]$ is to apply the general 
deformation theory of Kodaira-Spencer on complex structures to compact Hermitian locally symmetric spaces $\Gamma \backslash X$. It gives vanishing results on cohomology groups with coefficients in the sheaf $\Theta$ of germs of holomorphic tangent vector fields. If $X$ is an irreducible bounded symmetric domain different from the unit disc in $\mathbb{C}$, the first cohomology group vanish, and hence $\Gamma \backslash X$ is infinitesimally rigid. The paper $[\mathrm{CaV}]$ also contains a result, related to [Se3, Theorem, p. 159], which states that if $\Gamma \backslash X$ is a compact irreducible Hermitian locally symmetric space and $X \neq \mathbb{H}^{2}$, and $G$ is the group of all holomorphic isometries of $X$, then under a suitable conjugation by elements of $G$, the matrix entries of elements of $\Gamma$ are contained in a number field.

[Ca] proves similar vanishing results on cohomology groups for compact locally symmetric spaces of constant sectional curvature. The methods used in these papers are of Bochner type, which have also been used in other geometric proofs of rigidity results, for example in the super-rigidity in [Co] and [GS] for rank one lattices acting on the quaternionic hyperbolic spaces and the hyperbolic Cayley plane.

The paper [We] proves the local (or deformation) rigidity of irreducible uniform lattices of semsimple Lie groups without simple factor of dimension 3 or compact simple factors, which was conjectured by Selberg [Se3, p. 161]. It also uses Bochner type arguments or rather arguments from [Ca].

After these local rigidity results on locally symmetric spaces, Mostow obtained global (or strong) rigidity results of locally symmetric spaces [Mos1]. One version of the Mostow strong rigidity states as follows:

Suppose that $G, G^{\prime}$ are connected semisimple Lie groups with trivial center and no compact factors, and $\Gamma \subset G$ and $\Gamma^{\prime} \subset G^{\prime}$ are two uniform lattices. If $\Gamma$ is irreducible and $G$ is not isomorphic to $P S L(2, \mathbb{R})$, then every isomorphism $\pi: \Gamma \rightarrow \Gamma^{\prime}$ extends to an isomorphism $\pi: G \rightarrow G^{\prime}$.

If we take $G=G^{\prime}$, this strong rigidity property of $\Gamma$ implies the local (deformation) rigidity of $\Gamma$ in $G$.

Another version can be stated in terms of locally symmetric spaces. Let $K \subset G$ and $K^{\prime} \subset G^{\prime}$ be maximal compact subgroups, and $X=G / K$ and $X^{\prime}=G^{\prime} / K^{\prime}$ be their associated symmetric spaces of noncompact type, endowed with invariant Riemannian metrics. Then the Mostow strong rigidity is equivalent the following result:

Suppose that $\Gamma \backslash X$ is irreducible and $X$ is not equal to the hyperbolic plane $\mathbb{H}^{2}$. If $\Gamma \backslash X$ and $\Gamma^{\prime} \backslash X^{\prime}$ are homotopic, then they are isometric when the metrics on $X$ and $X^{\prime}$ are suitably scaled on their reducible factors. 
This formulation is closely related to the Borel conjecture in geometric topology: If two closed aspherical manifolds $M$ and $N$ are homotopic, then they are homeomorphic.

By definition, $M$ is an aspherical manifold if $\pi_{i}(M)=\{1\}$ for all $i \geq 2$. Locally symmetric spaces of noncompact type are aspherical manifolds, and the Mostow strong rigidity settles this conjecture when both $M$ and $N$ are locally symmetric spaces.

The Borel conjecture is related to classification of manifolds and was directly motivated by an earlier result of Mostow on solvmanifolds. Though many important cases have been proved, it is not completely solved yet and is a major unsolved problem in geometric topology. See [Fa] and references there (also [Ji1] for more references).

In the above version of the Mostow strong rigidity, the lattices $\Gamma$ are assumed to be co-compact. A natural problem is to remove this condition. This was achieved by Margulis in [75-2] when the rank of $G$ is at least two, and the proof was totally different from the remarkable proof of Mostow of strong rigidity of uniform lattices [Mos1] and was more in the style of the theory of automorphisms of classical groups. The rank one case was proved by Prasad [Pr1] by generalizing the method of [Mos1]. (See [Kos] and the introduction of [Pr1] for the history of the Mostow strong rigidity for non-uniform lattices). Therefore the strong rigidity holds for all irreducible lattices in semisimple Lie groups with trivial centers which are not locally equal to $S L(2, \mathbb{R})$ and have no compact factors.

In an earlier paper [Mos2, §12], Mostow proved the following result: Let $G$ be the group of isometries of a real hyperbolic space $X$ of dimension $n \geq 3$, and $\Gamma, \Gamma^{\prime}$ be two lattice subgroups of $G$. Let $\theta: \Gamma \rightarrow \Gamma^{\prime}$ be an isomorphism and $\varphi: X \rightarrow X$ a quasi-conformal homeomorphism such that

$$
\varphi(\gamma x)=\theta(\gamma) \varphi(x)
$$

for all $\gamma \in \Gamma$ and $x \in X$. Then $\theta$ extends to an inner automorphism of $G$. In particular, $\Gamma \backslash X$ and $\Gamma^{\prime} \backslash X$ are isometric.

A corollary of this result is the following: Assume that $M$ and $N$ are two compact Riemannian $n$-manifolds with constant negative curvature and $n>2$. If $M$ and $N$ are diffeomorphic, then they conformally equivalent and hence isometric.

In [Mos2], the above assumption that $M$ and $N$ are diffeomorphic is important. In [70-1], Margulis strengthened this latter result and proved the following result: if two compact hyperbolic manifolds $M$ and $N$ of dimension at least 3 are homotopic, then they are isometric.

The methods and ideas of this two pages long paper [70-1] are much more important, though many people might not be aware of this fact. As it is well-known 
to many people, the important notion of quasi-isometries and the method of pushing things, for example, an equivariant homotopy equivalence, to the boundary at infinity of symmetric spaces of noncompact type are used crucially in the famous proof of the Mostow strong rigidity by Mostow in [Mos1], and have motivated a lot of recent developments in various subjects, in particular in geometric group theory. It is also well-known that Gromov revolutionized the study of finitely generated groups by putting on word metrics on them and approximating the universal covering of a compact manifold by the fundamental group with a word metric and using the idea of quasi-isometries. In this short paper [70-1], these important notions and methods were introduced for real hyperbolic spaces and discrete groups acting on them independently of [Mos1] (the methods used in [Mos1] were outlined in an ICM talk by Mostow in 1970 [Mos3]) and ahead of Gromov's work, for example, the famous papers [Gr2] [Gr3].

\section{Super-Rigidity of LATtices}

There have been a lot of extension and generalizations of the Mostow strong rigidity. One of the most significant is the super-rigidity of lattices by Margulis [75-4] [84-1] [91-1]. Many results were announced in [75-4] and some proofs were also outlined. The paper [84-1] was written in mid-1970s and appeared as an appendix to the Russian translation of [Rag1].

The history and motivations of the superrigity of irreducible lattices of higher rank semisimple Lie groups canbe best explained by a recollection of Margulis:

"In the late sixties I learnt about Mostow's fundamental work on strong rigidity. Thinking about it, I at some point realized that it would be possible to prove the arithmeticity of uniform higher rank lattices if one could prove a statement which is now called superrigidity. I believe (and this was confirmed by Mostow) that the superrigidity was a new phenomenon which had not been discovered before. The first proof of superrigidity was based on combination of methods from ergodic theory and algebraic group theory, and one of the important ingredients was Oseledec multiplicative ergodic theorem. ${ }^{8}$

One of the consequences of superrigidity is the classification (in a certain sense) of finite-dimensional representations of higher rank lattices and $S$-arithmetic groups. The reduction of this classification to superrigidity is based on the argument which is dual (again in a certain sense) to the famous "unitary trick" of H.Weyl. Let me be a little bit more precise. Roughly speaking, the superrigidity describes representations $\rho$ of a lattice $\Gamma$ with non-compact image. But in general this image

\footnotetext{
${ }^{8}$ Borel in his paper [Bo3, pp. 10, §7] wrote that "The work of Margulis [on arithmeticity of irreducible lattices of higher rank semisimple Lie groups] was based on a new principle, soon christened "superrigidity" by Mostow..."
} 
can be compact. In order to make it non-compact, one has to apply Galois automorphisms of $\mathbb{C}$ over $\mathbb{Q}$ to matrix coefficients of elements from $\rho(\Gamma)$; the desired non-compactness can be achieved if $\rho(\Gamma)$ is not finite.

It looks strange now, but when I worked on superrigidity I was not influenced by Furstenberg's work just because I was essentially not familiar with it. It is indeed strange because many ideas and methods introduced by Furstenberg are very similar in style to what I used. I learnt about Furstenberg's work only around 1974 and his boundary theory influenced me very much. In particular my proof of the normal subgroup thoerem could not exist without that theory. Another important ingredient in the proof was the use of Kazhdan's property (T). I should say that I consider the proof of the normal subgroup theorem as my best proof."

For simplicity, we only state one version of the super-rigidity for lattices in real Lie groups: Let $\mathbf{G}$ be a connected semisimple linear algebraic Lie group defined over $\mathbb{R}$ of $\mathbb{R}$-rank at least 2. Assume that the real locus $\mathbf{G}(\mathbb{R})$ has no compact factors. Denote the identity component of $\mathbf{G}(\mathbb{R})$ by $\mathbf{G}(\mathbb{R})^{0}$ and let $\Gamma \subset \mathbf{G}(\mathbb{R})^{0}$ be an irreducible lattice. Suppose that $k$ is a locally compact field of characteristic 0 , and $\mathbf{G}^{\prime}$ is a connected algebraic group defined over $k$, almost simple over $k$. For every homomorphism $\pi: \Gamma \rightarrow \mathbf{G}^{\prime}(k)$ with the image $\pi(\Gamma)$ being Zariski dense, the following holds:

(1) If $k=\mathbb{R}$ and $\mathbf{G}^{\prime}(\mathbb{R})$ is non-compact, then $\pi$ extends to a rational homomorphism of algebraic groups $\pi: \mathbf{G} \rightarrow \mathbf{G}^{\prime}$ defined over $\mathbb{R}$, in particular, extends to a Lie group homomorphism $\pi: \mathbf{G}(\mathbb{R}) \rightarrow \mathbf{G}^{\prime}\left(\mathbb{R}^{\prime}\right)$.

(2) If $k=\mathbb{C}$, then either the closure $\overline{\pi(\Gamma)}$ in the regular topology of the complex variety $\mathbf{G}^{\prime}=\mathbf{G}^{\prime}(\mathbb{C})$ is compact, or the homomorphism $\pi$ extends to a homomorphism of algebraic groups $\pi: \mathbf{G} \rightarrow \mathbf{G}^{\prime}$.

(3) If $k$ is a totally disconnected local field such as $\mathbb{Q}_{p}$, then the closure of $\pi(\Gamma)$ in the locally compact space $\mathbf{G}^{\prime}(k)$ is compact.

In the above statement, the assumption that the image $\pi(\Gamma)$ is Zariski dense in $\mathbf{G}^{\prime}$ can be removed. In fact, it was shown by Margulis that the Zariski closure of $\pi(\Gamma)$ is a semisimple (or rather reductive) algebraic group, and hence projecting to suitable factors reduces to the previous case. For the most general version of the super-rigidity of lattices, see [75-3, Theorems 6,8$]$ [91-1, Theorem 6.15 of Chapter IX].

The Mostow strong rigidity is a special case of the above Margulis superrigidity when $k$ is required to be either $\mathbb{R}$ or $\mathbb{C}$, and the image $\pi(\Gamma)$ is a lattice in $\mathbf{G}^{\prime}(k)$. (Note that by the Borel density, lattices are Zariski dense). In a sense, the Margulis super-rigidity classifies finite dimensional representations of irreducible lattices $\Gamma$ of $\mathbf{G}(\mathbb{R})$ in terms of finite dimensional representations of algebraic groups $\mathbf{G}$, which are understood quite well. 
A more important application of the Margulis super-rigidity is to prove arithmeticity of such lattices $\Gamma$. In fact, as Selberg observed in [Se3, p. 159, p. 164], if a lattice $\Gamma$ in a linear semisimple Lie group $G$ is locally rigid, then under a suitable conjugation by elements of $G$, the matrix entries of elements of $\Gamma$ belong to a number field, hence belong to $\mathbb{Q}$ when embedded into a bigger general linear group by applying the functor of restriction of scalars. By the local rigidity of Weil [We] (or applying the stronger Mostow-Margulis-Prasad strong rigidity), an irreducible lattice $\Gamma$ in a connected semisimple Lie group $G$ with trivial center (hence linear) and no compact factors is locally rigid and thus admits a realization by matrices with entries contained in $\mathbb{Q}$. But this does not imply that $\Gamma$ is an arithmetic subgroup yet, though it is suggestive (see [PS2, p. 189, paragraph $5]$ ). The remaining difficult step is to show that the denominators of the entries for all the elements of $\Gamma$ are uniformly bounded. This is equivalent to the fact that they are bounded in the $p$-adic topology, which is settled by the Margulis super-rigidity when $k$ is taken to be a locally compact, totally disconnected field such as $\mathbb{Q}_{p}$. (See [Zi, p. 120-121] [91-1, Chap. IX, §2] for details.)

Since there are non-arithmetic lattices acting on the real hyperbolic spaces of all dimensions and complex hyperbolic spaces of dimensions less than or equal to 3, the Margulis super-rigidity can not be true for these lattices in $P S O(n, 1)$ and $\operatorname{PSU}(n, 1)$. For the other two rank 1 symmetric spaces, the quaternionic hyperbolic spaces and the hyperbolic Cayley plane, lattices acting on them do satisfy super-rigidity properties. Over Archimedean fields $\mathbb{R}$ and $\mathbb{C}$, it was proved by Corlette [Co], and the super-rigidity over $p$-adic fields was proved by Gromov and Schoen [GS]. In both cases, harmonic maps and Bochner type arguments are used. Other references on geometric super-rigidity include [JoY1-2] [MSY], where the super-rigidity is proved for uniform lattices and some non-uniform lattices.

See [Ji1-2] for more references on and some expositions of differential geometric proofs of Mostow-Margulis rigidity of locally symmetric spaces and generalizations to Kähler manifolds, in particular, the Siu-Yau method of proving rigidity results using harmonic maps.

\section{Normal SUbGroups of LATtices}

As mentioned before, one of the motivations for Selberg to make the conjecture on arithmeticity of lattices is to get a better understanding of the fundamental domains of such lattices on symmetric spaces, in order to develop the spectral theory of automorphic forms and the Selberg trace formula. Another is to obtain group structures of such lattices.

The results of Margulis super-rigidity and arithmeticity of lattices and methods developed to prove them have many other striking applications on understanding intrinsic structures of such lattices. 
Let $\Gamma$ be an irreducible lattice in a connected semisimple Lie group $G$ with finite center and no compact factors. Assume that the rank of $G$ is at least 2 . Then a known theorem of Kazhdan implies that the quotient $\Gamma /[\Gamma, \Gamma]$ is finite (see [Zi, Corollary 7.1.10, p. 132]). Clearly, $[\Gamma, \Gamma]$ is an infinite normal subgroup of $\Gamma$. Motivated by this and a result of Vaserstein [Vas], Margulis asked [75-4, p. Problem 3] if any infinite normal subgroup of $\Gamma$ has finite index. This is completely settled by him in [78-1] [78-2] [79-1]. Briefly, the results can be summarized as follows: Let $G$ be a connected semisimple Lie group with no compact factors and finite center and of rank at least 2, and $\Gamma \subset G$ an irreducible lattice. For every normal subgroup $N \subset \Gamma$, the quotient $N \backslash \Gamma$ has property $T$ of Kazhdan. If $N \backslash \Gamma$ is amenable, then it is finite, i.e., $N$ is of finite index; if $N \backslash \Gamma$ is not amenable, then $N$ is contained in the center $Z(\Gamma)$ of $\Gamma$ and hence is finite.

Roughly, this result says that such an irreducible lattice is simple modulo finite groups. If the irreducible lattice can be realized as an arithmetic subgroup of a linear semisimple algebraic group $\mathbf{G}$ defined over $\mathbb{Q}$, then $\mathbf{G}$ is almost simple over $\mathbb{Q}$. This brings out another close relation (or similarity) between the algebraic group $\mathbf{G}$ and its lattice subgroup $\Gamma$. It also reminds one of the famous construction of finite simple groups of Lie type from Chevalley groups.

The normal subgroup theorem is extremely elegant and can stand alone as a significant result. Naturally, there have been many applications of it. According to Margulis,

"One of main applications of the normal subgroup theorem is the statement about the finiteness of index of every non-central normal subgroup of a $S$-arithmetic group (under certain conditions). When $S$ is finite, this is a direct consequence of the normal subgroup theorem (in combination with the Borel-Harish-Chandra theorem). To go from finite $S$ to infinite $S$, one has to use the strong approximation for semi-simple groups over global fields. This reduction was first noticed by Gopal Prasad."

Another unexpected deep result related to ideas of Margulis and this normal subgroup theorem is the construction by Burger and Mozes [BuM1] of new examples of infinite simple groups satisfying the following conditions: (1) finitely presented, (2) torsion-free, (3) equal to fundamental groups of finite, locally CAT(0)-complexes, (4) of cohomological dimension 2, (5) biautomatic and (6) equal to the free amalgams of two isomorphic free groups over a common finite index subgroup.

These new groups are very different from all the known examples of finitely presented simple groups and answer positively several questions posted by various people. They arise as lattices in products of automorphism groups of regular trees, which are new classes of locally compact groups, for example, are not Lie groups. Briefly, they are given by certain lattices of Aut $T_{1} \times$ Aut $T_{2}$ whose projections 
in each factor satisfy various transitivity conditions, in particular, the so-called locally quasiprimitive condition [BuM2]. Another new feature of such lattices in comparison with lattices in semisimple Lie groups is that that cocompact lattices in Aut $T_{1} \times$ Aut $T_{2}$ never have dense projections in the two factors.

Briefly, Burger and Mozes give a criterion for non-residually finiteness of cocompact irreducible lattices $\Gamma$ of Aut $T_{1} \times$ Aut $T_{2}$, and also prove that irreducible lattices in Aut $T_{1} \times$ Aut $T_{2}$ satisfies the analogue of the Margulis normal subgroup theorem. Using these results, the new class of simple infinite groups can be constructed from non-residually finite cocompact lattices of Aut $T_{1} \times$ Aut $T_{2}$.

Another type of simple infinite groups are recently obtained from lattices in minimal Kac-Moody groups by Caprace and Remy [CaR], where the analogue of the Margulis normal subgroup theorem is also used crucially.

A further major application of the Margulis normal subgroup theorem is the proof of the following result by Polterovich [Pol, Corollary 1.1.D]: Let $\Gamma$ be irreducible non-uniform lattice in a connected semisimple real Lie group $G$ of real rank at least two without compact factors and with finite center. Then every homomorphism from $\Gamma$ into the group of smooth symplectic diffeomorphisms of a compact oriented surface of genus at least two has finite image.

In the proof of the Margulis normal subgroups, the theorem on factors of boundary actions plays a crucial role (see [91-1, Theorems 4.5 and 4.8 of Chapter IV] and [91-1, Theorem 4.3 of Chapter VI]). As mentioned in the previous section, Margulis considered the proof of the normal subgroup theorem as his best proof. For other developments on these results, see papers by Nevo and Zimmer [NeZ12], Dani [Da4], and Bader and Shalom [BaS].

\section{NORMAL SUbGROUPS OF GROUPS OF RATIONAL POINTS AND Margulis-Platonov CONJECTURES}

Let $\mathbf{G}$ be a simple simply connected algebraic group defined over a number field $k$. Let $\mathbf{G}(k)$ be its group of $k$-rational points. A natural problem is to understand normal subgroups of $\mathbf{G}(k)$.

One result in [78-2] says that every noncentral normal subgroup of $\mathbf{G}(k)$ has finite index. In particular, the commutator subgroup $[\mathbf{G}(k), \mathbf{G}(k)]$ has finite index in $\mathbf{G}(k)$. This is also proved by Prasad in [Pr2, Theorem C, p. 569].

One basic question is to understand conditions under which $\mathbf{G}(k)$ has no noncentral proper normal subgroups, i.e., $\mathbf{G}(k)$ is projectively simple.

A conjecture of Platonov states that $\mathbf{G}(k)$ is projectively simple if and only if for all places $\mu$ of $k$, the local groups $\mathbf{G}\left(k_{\mu}\right)$ are projectively simple. 
If $\mathbf{G}(k)$ is not projectively simple, a conjecture of Margulis in [78-2] describes its noncentral normal subgroups. See [PIR, Chapter 9] for precise statements and a survey of some results on these conjectures. For more recent results, see [Seg] [RaSS].

In [80-1], Margulis proved a related conjecture of Kneser on noncentral normal subgroups of multiplicative groups of a quaternion algebra over a global field.

\section{Characterization of arithmetic groups}

As mentioned earlier, one of the motivations of the Selberg conjecture on arithmeticity of lattices is to get a better understanding of the geometry at infinity of locally symmetric spaces. Another reason is that if $\Gamma$ is an arithmetic subgroup of a linear semisimple algebraic group $\mathbf{G}$ defined over $\mathbb{Q}$, then the associated locally symmetric space $\Gamma \backslash X$ has a lot of symmetry.

The isometry group of $\Gamma \backslash X$ as a Riemannian manifold is finite, as a special case of finiteness of the isometry group of a complete nonpositively Riemannian manifold of finite volume. But it admits many (Hecke) correspondences, which are important for understanding various problems on $\Gamma \backslash X$, for example, the Euler product of automorphic functions, and the spectral theory of automorphic forms, in particular a conjecture of Sarnak on abundance of cuspidal eigenfunctions (the Weyl law of the counting function of the cuspidal eigenvalues, to be precise) for noncompact arithmetic locally symmetric spaces [Sar1].

More specifically, define the commensurability subgroup of $\Gamma$ in $\mathbf{G}$ by:

$$
\operatorname{Comm}(\Gamma)=\left\{g \in \mathbf{G}(\mathbb{R}) \mid g \Gamma g^{-1} \text { and } \Gamma \text { are commensurable }\right\}
$$

Then every element $g \in \operatorname{Comm}(\Gamma)$ induces a correspondence on $\Gamma \backslash X$. Clearly $\Gamma \subset \operatorname{Comm}(\Gamma)$. It can be shown easily that $\mathbf{G}(\mathbb{Q}) \subset \operatorname{Comm}(\Gamma)$ (Note that $\Gamma$ is assumed to be arithmetic). If the algebraic group $\mathbf{G}$ has trivial center and the real locus $\mathbf{G}(\mathbb{R})$ has no compact factors, then $\operatorname{Comm}(\Gamma)$ is dense in $\mathbf{G}(\mathbb{R})$, and $\mathbf{G}(\mathbb{Q})=\operatorname{Comm}(\Gamma)$.

In [75-4, Theorem 9], Margulis proved that if $\Gamma$ is an irreducible lattice in a connected semisimple Lie group with no compact factors and trivial center, then $\Gamma$ is arithmetic if and only if $\operatorname{Comm}(\Gamma)$ is dense in $G$. This characterization of arithmetic subgroups was conjectured by Piateski-Shapiro and Shafarevich [PS1]. (For characterization of arithmetic Fuchsian groups, see the references in [Ji1]). 


\section{FURTHER DEVELOPMENTS ON RIGIDITY OF LATTICES}

There have been many extensions and generalizations of results related to the themes discussed in the previous sections: arithmeticity, strong rigidity and super-rigidity of lattices of semisimple (real and $p$-adic) Lie groups. ${ }^{9}$

Both semisimple real Lie groups and $p$-adic Lie groups are related to semisimple algebraic groups over fields of characteristic 0 , and a natural generalization is to prove super-rigidity and arithmeticity of lattices in high rank semisimple groups over local fields of arbitrary characteristic. Such results have been proved by Venkataramana [Ve]. Another generalization is to consider arithemticity of lattices in adelic groups, which was proved by $\mathrm{Oh}$ in [Oh3].

As discussed above, Margulis considered both real and $p$-adic semisimple Lie groups of higher rank, and arithmeticity and super-rigidity of lattices in them. For related results on characterization of rank one lattices of groups in positive characteristic, see [Li1] (see also [Li2] for related results on super-rigidity of lattices over positive characteristic).

Note that lattices of semisimple real Lie groups act on symmetric spaces of non-compact type, and lattices of semisimple $p$-adic Lie groups act on BruhatTits buildings, and rigidity properties of lattices of semisimple Lie groups can be studied in terms of these actions.

Both symmetric spaces of non-compact type and Bruhat-Tits buildings are CAT(0)-spaces. But there are many other natural CAT(0)-spaces, for example, products of trees. Therefore, it is natural to study discrete group actions on CAT(0)-spaces and their rigidity properties analogous to the strong rigidity and super-rigidity of irreducible lattices of semisimple (real and $p$-adic) Lie groups. Similarly, it is also natural to study rigidity properties of group actions on CAT(1)-spaces, which correspond to rank 1 symmetric spaces of non-compact type and include trees as important examples.

Furthermore, instead of replacing Lie groups by the automorphism groups of CAT(0)-spaces and of CAT(-1)-spaces as in the previous paragraph, one could also consider locally compact, non-discrete, compactly generated groups and their products, and discrete subgroups contained in them, and try to generalize important properties of irreducible lattices in semisimple Lie groups such as the normal subgroup theorem etc..

\footnotetext{
${ }^{9}$ It should be emphasized that there have been many significant results in these directions, and the rather incomplete and brief summary of literature in this short section only represents the limited knowledge of the author of this article and includes those brought to his attention by various experts.
} 
The paper [06-3] proves a quite general super-rigidity theorem for actions of irreducible lattices in products of locally compact groups on Busemann nonpositively curved uniformly convex metric spaces and also includes a proof of an unpublished result of Margulis on commensurability super-rigidity in a general setting.

See also [Bur] [Moz1] [BaS] [Fur] [Mon] [Gao] for summaries and references on these topics and related results.

Another important generalization of the Mostow and Margulis rigidity theory is the Zimmer program on rigidity properties and classification of actions of higher rank semisimple Lie groups, their irreducible lattices actions on compact manifolds preserving various geometric structures, and orbit equivalence rigidity. In certain sense, the Zimmer program is a non-linear generalization. A direct analogue of the Margulis super-rigidity is the Zimmer cocycle super-rigidity. See [Zi1] [Zi2] for details. See also the recent surveys [Pop] and [Sh] on related topics.

\section{Counting Lengths of Geodesics And volume Entropy, And MARGULIS MEASURE}

If $M$ is a compact Riemannian manifold with strictly negative sectional curvature, then each homotopy class of closed curves contains a unique closed geodesic, and the lengths of closed geodesics in $M$ form an increasing sequence with finite multiplicity and going to infinity. A natural problem is to understand asymptotic behaviors of the counting function of this sequence.

One result of Margulis' thesis, parts of which were published in [69-2] [70-2], and an English translation in its entirety was recently published in [04-1], gives the leading term of such asymptotics.

Specifically, denote the counting function of the lengths of closed (prime) geodesics $\gamma$ in $M$ by $\pi(T)$ :

$$
\pi(T)=\mid\{\gamma \mid \ell(\gamma) \leq T, \text { where } \gamma \text { is a closed prime geodesic in } M\} \mid .
$$

Let $h$ be the volume entropy of $M$, i.e., the exponential growth rate of the volume of balls $B_{\tilde{M}}\left(R, x_{0}\right)$ in the universal covering $\tilde{M}$ of $M$ as the radius $R \rightarrow+\infty$. Then as $T \rightarrow+\infty$,

$$
\pi(T) \sim \frac{e^{h T}}{h T}
$$

This is similar to the prime number theorem counting prime integers and hence is called the generalized prime number theorem. Briefly, the prime numbers can be studied by the Riemann zeta function through the Euler product. For a compact Riemann surface with hyperbolic metric $\Gamma \backslash \mathbb{H}$, there is also a Selberg zeta function, which can also be expressed as products over prime closed geodesics of $\Gamma \backslash \mathbb{H}$. This 
Selberg zeta function can be used to obtain the asymptotic of $\pi(T)$, i.e., the generalized prime number theorem in this case. Since the Poisson summation formula is used to study the Riemann zeta function and the Selberg trace formula for $\Gamma \backslash \mathbb{H}$ is a non-abelian analogue of the Poisson summation formula and is used to study the Selberg zeta function, the generalized prime number theorem for geodesics is a non-abelian generalization of the usual prime number theorem counting prime integers. For more discussions of the special case of $\Gamma \backslash \mathbb{H}$, see the book [Hej].

Closed geodesics in $M$ correspond to periodic orbits of the geodesic flow on $M$ (or rather tangent bundle, or its unit sphere). It is known that when the sectional curvature is strictly negative, the geodesic flow is Anosov. Margulis's thesis also studies general Anosov systems and gives asymptotic properties of lengths of periodic orbits and other invariants. An important tool in this study is the so-called Margulis measure.

Indeed, the Margulis measure (also called the Bowen-Margulis measure) for an Anosov flow is the unique flow-invariant probability measure of maximal entropy and has played an important role in ergodic theory, and its construction by Margulis is another major result of Margulis' thesis. It was first published in [70-2] before the full version [04-1]. For more details about this measure, relations to different notions of entropy, and applications, see also the book $[\mathrm{KaH}]$.

This thesis of Margulis has motivated several active areas of research. Besides counting length distribution of closed geodesics, another aspect of the thesis [041] of Margulis concerns distribution (or counting) of orbits of discrete groups acting co-compactly on a simply connected strictly negatively curved manifold, in particular, a rank one symmetric space of non-compact type. Counting orbits of discrete groups is an important and natural problem, for example, when the group is $\mathbb{Z}^{n}$ acting on $\mathbb{R}^{n}$ by the standard action or through a linear representation, then it is the classical circle problem on integral points. For some generalization of this result to higher rank symmetric spaces of non-compact type, see [GO].

There is also a zeta function which encodes the lengths of periodic orbits. See the survey article by M.Sharp in [04-1] for developments after this marvelous thesis. See also the review of [O4-1] by Parry [Par].

\section{LONG TIME BEHAVIOR OF UNIPOTENT GROUP ORBITS}

When a Riemannian manifold $M$ is given by a rank 1 locally symmetric space $\Gamma \backslash X$, geodesics correspond to orbits of the Cartan subgroup $A$ of $G$ in the homogeneous space $\Gamma \backslash G$.

If we replace the subgroup $A$ by a one-parameter subgroup of unipotent elements $\left\{u_{t}\right\}$ of $G$, we get a unipotent flow on homogeneous spaces of $G$. Unipotent 
flows and actions of unipotent subgroups on homogeneous spaces have been studied in many papers of Margulis. They played an important role in his proof of the arithmeticity of non-uniform lattices of higher rank semisimple Lie groups and his solution of Oppenheim conjecture on values of irrational indefinite quadratic forms at integral points.

In [71] [75-3], Margulis proved the following result: Let $G=G L(n, \mathbb{R})$. For any one-parameter group $\left\{u_{t}\right\}$ of unipotent linear transformations, the orbit of every point in the homogeneous space $G L(n, \mathbb{R}) / G L(n, \mathbb{Z})$ under the semi-group $\left\{u_{t}\right\}, t \geq 0$, does not diverge to infinity. This result was conjectured by PiateskiShapiro and was used by Margulis to prove arithmeticity of higher rank lattice subgroups of semisimple Lie groups.

If an orbit does not diverge to infinity, a natural question is how often it returns to compact regions. Such a result was proved by Dani and Margulis in [91-3], following earlier work of Dani [Da1] [Da2]. Let $\mathbf{G}$ is a semisimple algebraic $\mathbb{Q}$ group and $\Gamma \subset \mathbf{G}(\mathbb{Q})$ be an arithmetic subgroup in $G=\mathbf{G}(\mathbb{R})$. Then there exists a compact subset $K$ of $G / \Gamma$ such that, for any unipotent one-parameter subgroup $\left\{u_{t}\right\} \subset G$ and any $g \in G$, the time spent in $K$ by the $u_{t}$-orbit of $g \Gamma$ during the time interval $[0, T]$ is asymptotic to $T$, unless $\left\{g^{-1} u_{t} g\right\}$ is contained in a $\mathbb{Q}$-parabolic subgroup of $\mathbf{G}$.

See the paper $[97-3, \S 3]$ for a summary of various improvements and related results.

Further significant improvements were obtained in a joint paper with Kleinbock [98-2] and the paper by Kleinbock [Kl], and generalizations to S-arithmetic cases were obtained by Kleinbock and Tomanov [KlT].

\section{EXPANDER GRAPHS AND WORK IN COMBINATORICS}

Probably most people have heard of the Margulis arithmeticity theorem of irreducible lattices in higher rank semisimple Lie groups, but some may not know that Margulis [73-2] was the first one who explicitly constructed expanders (or expanding graphs). For a comprehensive discussion of the topics in this and the next section, see the books [Lu3] [Sar2].

Roughly, an expander is a finite graph with few vertices but high connectivity properties, i.e., many edges from all vertices. Specifically, let $c>0$ and $k, n$ be positive integers. A $k$-regular graph $X$ with $n$-vertices is called a c-expander if for every subset $A$ of $X$, the following isoperimetric type inequality holds:

$$
|\partial A| \geq c\left(1-\frac{|A|}{n}\right)|A|,
$$

where the boundary is $\partial A=\{y \in X \mid d(y, A)=1\}, d(\cdot, \cdot)$ is the distance function on $X$ with every edge length equal to 1 . 
Clearly, every finite ( $k$-regular) graph admits a positive $c$ satisfying the above condition. But the point is to find a uniform constant $c$ which works for an infinitely family of $k$-regular graphs.

Expanders are useful for many purposes and are sought after by people in several fields. Random graphs are expanders, but it was not easy to construct them explicitly, and hence they are difficult to implement for practical applications. In [73-2], Margulis used groups with property T of Kazhdan to construct expanders. Many other constructions followed.

On a connected compact manifold $M$ without boundary, the constant functions are eigenfunctions of the Laplace operator with eigenvalue equal to 0 , which has multiplicity 1. The next eigenvalue $\lambda_{1}(M)$ is positive and is related to an isoperimetric invariant, called the Cheeger constant, of the manifold. In fact, it basically follows from the characterization of eigenvalues in terms of the Rayleigh quotients. These notions can be generalized to graphs.

For graphs, the Cheeger constant is comparable to the expanding constant $c$ in Equation (1). Therefore, the key point in constructing expander graphs is to find graphs on which the eigenvalue of constant eigenfunctions is isolated by a definite amount from other eigenvalues. This exactly corresponds to the property $\mathrm{T}$ of Kazhdan, for which the trivial representation is isolated by a definite amount from other unitary representations in the unitary dual of the group. See [Lu3, $\S 3.3]$ (also [Zi1]) for definition of property $\mathrm{T}$ and related results.

In [88-1], Margulis used the positive solution of Ramanujan conjecture for modular forms or automorphic representations, which is also basically related to a definite isolation of the trivial representation in a regular representation, to construct expanders. This work is similar to the work of Lubotzky-PhillipsSarnak [LuPS].

Besides the above now well-known work on expanders by Margulis, his other work in combinatorics are equally deep and beautiful.

For a connected graph, an important invariant is the girth, which is the shortest length of cycles in it. The paper [82-1] constructs explicitly graphs with large girths. For graphs whose vertices have degree greater than or equal to three, it can be shown easily that the girth grows at most at the rate of the logarithm of the number of vertices. It is not difficult either to prove the existence of such graphs whose girth has the maximal growth rate, i.e., it is a logarithmic function of the number of vertices. But it is difficult to explicitly construct them. A basic result of [82-1] gives an explicit construction of such graphs with maximal girth growth. It should be emphasized that these graphs are the first explicitly constructed such graphs. As an application, it also gives an explicit construction of a sequence of low density codes for which the probability of errors of decoding tends to zero. The paper [84-4] contains related results. 
The paper [74-3] deals with a sharp transition behavior of the probability $f(p)=f_{G}(p)$ such that a connected graph $G$ becomes disconnected when every edge is deleted with probability $p$.

Clearly, $f(p)$ is an increasing function of $p$ with $f(0)=0$ and $f(1)=1$. For $\varepsilon \in[0,1]$, define

$$
t_{1}(\varepsilon)=\inf \{p \mid f(p) \geq \varepsilon\},
$$

which is the cut-off point for $f(p)$ lying below $\varepsilon$,

$$
t_{2}(\varepsilon)=\sup \{p \mid f(p) \leq 1-\varepsilon\},
$$

which is the cut-off point for $f(p)$ lying above $1-\varepsilon$, and

$$
t(\varepsilon)=t_{2}(\varepsilon)-t_{1}(\varepsilon)
$$

which is the transition gap in $p$ for $f(p)$ to change from $\varepsilon$ to $1-\varepsilon$. The main result of this paper [74-3] states that for all $\varepsilon, \delta \in[0,1]$, there exists an integer $n=n(\varepsilon, \delta)$ such that

$$
t(\varepsilon)<\delta
$$

for every $n$-connected graph. (Recall that a graph $G$ is said to be $n$-connected if there does not exist a set of $(n-1)$ vertices whose removal disconnects the graph, i.e., its complement in $G$ is disconnected. For example, a connected graph is 1-connected, and a biconnected graph is 2-connected.)

This bound on $t(\varepsilon)$ shows a sharp transition of $f(p)$ from being almost surely connected to being almost surely disconnected after edges are deleted with probability $p$. This reminds one of a similar sharp transition in the important pecolation theory (see the article by Kesten $[\mathrm{Ke}]$ and references there for more detail about percolation theory).

\section{INVARIANT MEASURES ON SPHERES AND $\mathbb{R}^{n}$}

Let $S^{n}$ be the unit sphere in $\mathbb{R}^{n+1}$ with the induced Riemannian metric. Then its associated Lebesgue measure $\lambda$ is countably additive and invariant under the totation group $O(n+1)$. It was shown by Lebesgue that $\lambda$ is the unique countably additive and rotation invariant measure on $S^{n}$ up to a scalar multiple. Banach showed that this uniqueness result fails for $n=1$ if the countable additivity is replaced by finite additivity. The famous Banach-Ruziewicz problem asks whether the uniqueness still holds for $n \geq 2$ if the countable additivity is replaced by finite addivitity, i.e., whether the Lebesgue measure $\lambda$ is the unique finitely additive measure defined on Lebesgue measurable subsets of $S^{n}$ which is invariant under $O(n+1)$ when $n \geq 2$.

It was finally solved positively for $n \geq 4$ by Margulis [80-2] and Sullivan [Sul1] by discrete groups having property $\mathrm{T}$ of Kazhdan, and for $n=2,3$ by Drinfeld 
[Dr] using the solution of the Ramanujan conjecture, all building on a crucial reduction step by Rosenblatt [Ro].

The basic idea of the solution of the Banach-Ruziewicz problem is as follows. First, any such finitely additive and invariant measure is absolutely continuous with respect to the Lebesgue $\lambda$ and hence can be used to integrate functions in $L^{\infty}\left(S^{n}\right)$, i.e., define a mean on $L^{\infty}\left(S^{n}\right)$. Then the Banach-Ruziewicz problem is equivalent to whether the Lebesgue integral on $L^{\infty}\left(S^{n}\right)$ is the only $S O(n+1)$ invariant mean on $L^{\infty}\left(S^{n}\right)$.

The subspace

$$
L_{0}^{2}\left(S^{n}\right)=\left\{f \in L^{2}\left(S^{n}\right) \mid \int_{S^{n}} f d \lambda=0\right\}
$$

is clearly invariant under $S O(n+1)$ and hence every finitely generated subgroup $\Gamma$. (It might be worthwhile to point out that this subspace is used to characterize the first positive eigenvalue of $S^{n}$ in terms of the Rayleigh quotients.)

The second step is that if the induced representation of $\Gamma$ on $L_{0}^{2}\left(S^{n}\right)$ does not weakly contain the trivial representation, then the Lebesgue mean (or integral) is the unique invariant mean on $L^{\infty}\left(S^{n}\right)$.

The third step is to find a finitely generated group $\Gamma \subset S O(n+1)$ which has property $\mathrm{T}$ and is dense. In fact, the density of $\Gamma$ implies that its action on $S^{n}$ is ergodic and hence does not have any invariant nonzero function in $L_{0}^{2}\left(S^{n}\right)$; and property $\mathrm{T}$ implies that its representation does not weakly contain the trivial representation.

The Lebesgue measure on $\mathbb{R}^{n}$ is also countably additive and invariant under the isometry group. The Banach-Ruziewicz problem can also be formulated for $\mathbb{R}^{n}$ : Is the Lebesgue measure the unique finitely additive isometry-invariant positive measure on $R^{n}$ up to a scalar multiple? The answer is no by Banach for $n=1,2$. In [82-2], Margulis gave a positive answer for $n \geq 3$.

A quantitive version of the Banach-Ruziewicz problem for measures on $S^{n}$ has also been obtained by Clozel [Cl] for even $n$ and $\mathrm{Oh}$ [Oh5] for odd $n$. For a new and elementary proof of the Ruziewicz problem on $S^{2}$ and other related results, see the paper by Gamburd, Jakobson and Sarnak [GaJS].

\section{Strong Approximation of ALGEBraic groups}

The strong approximation is an important arithmetic property of linear algebraic groups defined over global fields. It is a generalization of the Chinese remainder theorem and can be formulated for all varieties defined over global fields. 
An important problem is to decide which varieties, or more restrictively linear algebraic groups, satisfy the strong approximation property. For general varieties, not much is known. On the other hand, for linear algebraic groups, it is settled. Unipotent linear groups and affine spaces satisfy the strong approximation, and the Levi decomposition reduces the problem to semisimple linear groups.

For semisimple and simply connected linear algebraic defined over number fields, the strong approximation was established by Kneser and Platonov (see [PlR, Chapter 7]). Over function fields, it was established for such algebraic groups by Margulis [77-1] and Prasad [Pr2].

Briefly, the strong approximation for such algebraic groups is the following statement: Let $k$ be a global field of arbitrary characteristic, and let $\mathbf{G}$ be a connected, simply connected, semi-simple linear algebraic group defined and almost simple over $k$. Let $\mathbb{A}$ be the adele ring of $k$ and let $S$ be a finite set of places of $k$. For each $\mu \in S$, let $k_{\mu}$ be the corresponding local field. Define

$$
G_{S}=\prod_{\mu \in S} \mathbf{G}\left(k_{\mu}\right)
$$

Assume that $G_{S}$ is noncompact. Then the subgroup $\mathbf{G}(k) G_{S}$ is dense in $\mathbf{G}(\mathbb{A})$.

The strong approximation is derived from the validity of the Kneser-Tits conjecture for algebraic groups over local fields and the following result. Let $K$ be a local field of arbitrary characteristic. Let $\mathbf{G}$ be a connected semi-simple algebraic group defined over $K$. Assume that $\mathbf{G}$ is isotropic and almost simple over $K$. Let $G=\mathbf{G}(K)$ be the group of $K$-rational points of $\mathbf{G}$. Denote by $G^{+}$the normal subgroup of $\mathbf{G}(K)$ generated by the $K$-rational points of the unipotent radicals of $K$-parabolic subgroups of $\mathbf{G}$. Then the main theorem in [77-1] [Pr2] states: Let $H$ be a closed non-discrete subgroup of $G$ such that $G / H$ carries a finite $G$-invariant Borel measure. Then $H \supset G^{+}$; in particular, $G / H$ is compact. If, moreover, $G$ is simply connected, then $H=G$.

\section{Maximal Subgroups of LATtices AND Linear GRoups}

The arithmetic group $S L(n, \mathbb{Z})$ is an important example of finitely generated group, and understanding its subgroups is a natural and important problem. The purpose of the joint papers of Margulis with Soifer [77-3] [79-2] [81-1] is to answer several questions about maximal subgroups of $S L(n, \mathbb{Z})$. One question of Platonov asks if $S L(n, \mathbb{Z})$ contains a maximal subgroup of infinite index.

To answer this question, the following general criterion is proved: Let $\Gamma$ be a finitely generated linear group over a field. Then all the maximal subgroups of $\Gamma$ have finite index if and only if $\Gamma$ has a solvable subgroup of finite index. This implies that for $n \geq 2, S L(n, \mathbb{Z})$ contains a maximal subgroup of infinite index. Margulis and Soifer also prove that if a finitely generated group over a field 
does not contain a solvable subgroup of finite index, then the set of its maximal subgroups of infinite index is uncountable.

The paper [79-2] [81-1] also answered negatively a question of Platonov and Prasad which asks if such a maximal subgroup of $S L(n, \mathbb{Z})$ of infinite index must contain a free group of finite index by proving the following result: When $n \geq 4$, $S L(n, \mathbb{Z})$ has a maximal subgroup of infinite index which contain a free abelian group of rank 2 and hence does not contain a free subgroup of finite index.

\section{DeComposition of LATtice SUBGroups into AMALGAMS}

An amalgam decomposition of a group $\Gamma$ is a free product with amalgamation. This is a basic and important operation in group theory. For example, it is known that every finitely generated infinite group has either 1, 2 or infinitely many ends. A famous theorem of Stalling [St] says that a torsion-free finitely generated group $\Gamma$ which has infinitely many ends is a free product with amalgamation.

Serre proved in his book [Ser] that a group $\Gamma$ acts on a tree without any common fixed point if and only if it either admits an amalgam decomposition or is an HNN extension, and every lattice of a rank-one simple algebraic group $\mathbf{G}$ over a local compact, totally disconnected field such as $\mathbb{Q}_{p}$ acts on the Bruhat-Tits building of $\mathbf{G}$, which is a tree, without common fixed points, and hence admits either an amalgam decomposition or is an HNN extension. If such a lattice is torsion-free, then it acts freely on the tree given by the Bruhat-Tits building, i.e., no point of the tree is fixed by any nontrivial element, and hence the lattice is a free group (see [Lu1] for more results on lattices in rank one Lie groups over local fields). Similarly, lattices in $\mathbb{R}$-rank-one simple real Lie groups such as $\mathrm{SL}_{2}(\mathbb{R}), \mathrm{SL}_{2}(\mathbb{C})$ also tend to admit amalgam decompositions. On the other hand, Serre observed that $\mathrm{SL}_{3}(\mathbb{Z})$ is not an amalgam product and conjectured that the same holds for higher rank irreducible lattices of semisimple Lie groups.

In [81-2], Margulis followed a suggestion of Serre and proved the following result using the method of equivariant measurable maps: If $\Gamma$ is an arithmetic subgroup of a connected, simply connected almost $\mathbb{Q}$-simple $\mathbb{Q}$-linear algebraic group with $\mathbb{R}$-rank $(\mathbf{G}) \geq 2$, then $\Gamma$ is not an amalgam product.

This conjecture is also proved by Watatani [Wa] and Alperin [Al]. In fact, they proved that if a countable discrete group $\Gamma$ has property $\mathrm{T}$ of Kazhdan, then $\Gamma$ has property FA of Serre, i.e., every action of $\Gamma$ on a tree has a global fixed point, which in turn implies that $\Gamma$ is not an amalgam product. One consequence is that such an arithmetic subgroup $\Gamma$ has only one end.

But the paper [81-2] gives a more general result as well as information about possible decompositions of $\Gamma$ when $\Gamma$ is an amalgam product. In fact, it proves that if $\Gamma$ is a lattice in a locally compact group $G$ which is a product of finitely 
many groups of type $\mathbf{G}_{\alpha}\left(k_{\alpha}\right)$, where $k_{\alpha}$ is a local field and $\mathbf{G}_{\alpha}$ a connected simply connected semisimple $k_{\alpha}$-linear algebraic group, then, under quite general conditions, any decomposition of $\Gamma$ as an amalgam product is induced by a decomposition of $G$ as an amalgam product of open subgroups. The proof is carried out by proving that an amalgamated action of $\Gamma$ on a tree can be extended to an action of $G$, similar to the extension of representations in the super-rigidity of irreducible higher rank lattices.

\section{Actions of affine transformations, Milnor conjecture, Auslander CONJECTURE}

The famous Auslander conjecture asks if every affine crystallographic group is virtually solvable. A stronger conjecture by Milnor requires only that $\Gamma \subset \operatorname{Aff}\left(\mathbb{R}^{n}\right)$ acting properly on $\mathbb{R}^{n}$, but without requiring compact quotients.

The papers [83-1] [84-2] disprove this conjecture of Milnor. Specifically, let $G$ be the product of $O^{0}(2,1)$ and the translation group of $\mathbb{R}^{3}, g_{1}, g_{2}$ be two distinct elements of $G$ such that the linear component of each of them fixes exactly two lines on the isotropic cone, and $\Gamma=\left\langle g_{1}, g_{2}\right\rangle$ be the subgroup generated by $g_{1}$ and $g_{2}$. Sufficient conditions are given for each of the following properties to hold:

(1) $\Gamma$ is freely generated by $g_{1}, g_{2}$,

(2) $\Gamma$ acts properly discontinuously on $\mathbb{R}^{3}$,

(3) $\Gamma$ does not act properly discontinuously on $\mathbb{R}^{3}$.

As a corollary, they show that $G$ contains a free subgroup $\Gamma$ on two free generators that acts properly discontinuously on $\mathbb{R}^{3}$. Clearly such a group $\Gamma$ is not virtually solvable. This space gives a counterexample to the Milnor conjecture and is called a Margulis space-time.

The joint paper with Grunewald [89-1] is also concerned with the Auslander conjecture. Specifically, let $A(n)$ be the group of affine motions on $\mathbb{R}^{n}$, i.e., the semidirect product $\mathbb{R}^{n} \rtimes G L_{n}(\mathbb{R})$, and $\ell: A(n) \rightarrow G L_{n}(\mathbb{R})$ be the canonical projection. For any $g \in A(n)$, the image $\ell(g)$ is called the linear part of the affine transformation of $g$. Suppose that $G$ is a closed subgroup with finitely many connected components in $G L_{n}(\mathbb{R})$. A group $\Gamma$ is called $G$-linear if $\ell(\Gamma) \subset G$. According to this definition, a subgroup of the Euclidean motion group $E(n)$ is then called $O(n)$-linear. Let $\operatorname{rk}_{\mathbb{R}}(G)$ be the real rank of $G$. One of the main results in [89-1] states that if $\operatorname{rk}_{\mathbb{R}}(G) \leq 1$, then any $G$-linear subgroup of $A(n)$ that acts properly discontinuously on $\mathbb{R}^{n}$ with compact quotient is virtually polycyclic.

The joint paper with Abels and Soifer [97-1] proves the Auslander conjecture for proper actions on $\mathbb{R}^{N}$ for all dimensions up to 6 , i.e., $N \leq 6$, by considering the algebraic hull (Zariski closure) of the linear holonomy group $l(\Gamma)$ in $G L(N, \mathbb{R})$, which are described as follows. 
Suppose that $\Gamma$ is a discrete group of affine transformations acting properly on $\mathbb{R}^{N}$. Then the following holds:

(1) If $N=4 m+1$, then $l(\Gamma)$ is not Zariski dense in a conjugate of $\mathrm{O}(2 m+$ $1,2 m)$

(2) if $N=4 m+3$, then there exists a free discrete group $\Gamma$ of affine transformations acting properly on $\mathbb{R}^{N}$ whose linear part is Zariski dense in $\mathrm{O}(2 m+2,2 m+1)$

(3) if $N=p+q$ with $|p-q| \neq 1$, then $l(\Gamma)$ is not Zariski dense in a conjugate of $\mathrm{O}(p, q)$.

The joint paper with Abels and Soifer [02-1] considers the question when the Zariski closure of the linear part is equal to the group $S O(n+1, n)$. The main results are as follows:

(1) When $n$ is even, there does not exist a properly discontinuous subgroup $\Gamma$ of $\operatorname{Aff}\left(\mathbb{R}^{2 n+1}\right)$ whose linear part is Zariski dense in $S O(n+1, n)$.

(2) When $n$ is odd, there exists a properly discontinuous free subgroup $\Gamma$ of Aff $\left(\mathbb{R}^{2 n+1}\right)$ whose linear part is Zariski dense in $S O(n+1, n)$.

The joint paper with Abels and Soifer [05-2] contains the following similar result: Assume that $B$ is a nondegenerate quadratic form of signature $(n-2,2)$ and let $O(B)$ denote the orthogonal group of the form $B$. Then the Auslander conjecture holds for all affine crystallographic groups satisfying $l(\Gamma) \subseteq O(B)$.

Further related results on affine actions are contained in [06-1].

\section{AbSence of invariant Analytic hypersurfaces.}

For $\Gamma$ a group acting on a complex space $X$, let $H(X)^{\Gamma}$ be the set of $\Gamma$ invariant analytic hypersurfaces of $X$, where an analytic hypersurface is a pure 1codimensional closed complex analytic subset. In 1981, Ahiezer [Ah] proved that if $G$ is a connected semisimple linear algebraic group defined over $\mathbb{Q}$, and $\Gamma \subset G_{\mathbb{Q}}$ is a Zariski dense discrete subgroup, then $H(G)^{\Gamma}=\varnothing$. This is related to the question that given a connected complex Lie group $G$, for which subgroups $\Gamma \subset G$, the field of meromorphic functions on $G$ invariant under the right translations by elements of $\Gamma$ contains only constants. In the joint paper with Huckleberry [83-2], it is proved that the converse of the result of Akhiezer is also true, i.e., if $G$ is a connected complex semisimple Lie group, and $\Gamma \subset G$ any subgroup, then $H(G)^{\Gamma}=\varnothing$ if and only if $\Gamma$ is Zariski dense in $G$. 


\section{Proportionality of Covolume of lattices}

Let $G$ be a semisimple Lie group and $\Gamma \subset G$ a lattice. Consider the set $\mathcal{A}_{\Gamma}$ of all lattice subgroups $\Gamma^{\prime}$ of $G$ commensurable with $\Gamma$. Fix a Haar measure on $G$. Then the volumes of all $\Gamma^{\prime} \backslash G, \Gamma^{\prime} \in \mathcal{A}_{\Gamma}$, are rational multiples of the volume of $\Gamma \backslash G$.

Let $X=G / K$ be the associated symmetric space with an invariant Riemannian metric. Then the volumes of locally symmetric spaces $\Gamma^{\prime} \backslash X$ are all rational multiples of the volume of $\Gamma \backslash X$ for $\Gamma^{\prime} \in \mathcal{A}_{\Gamma}$. When $X$ is the real three dimensional hyperbolic space $\mathbb{H}^{3}$, Thurston raised a question whether there is a number $v_{0}$ which depends on $\Gamma$ such the volume of every $\Gamma^{\prime} \backslash \mathbb{H}^{3}, \Gamma^{\prime} \in \mathcal{A}_{\Gamma}$, is an integral multiple of $v_{0}$. This is clearly true if there exists a unique maximal lattice in $\mathcal{A}_{\Gamma}$. But this assumption is often not true.

This problem of Thurston was solved by Borel [Bo1] for more general lattices in $G=P G L_{2}(\mathbb{R})^{a} \times P G L_{2}(\mathbb{C})^{b}, a, b \in \mathbb{N}, a+b \geq 1$. He also showed that when $\Gamma$ is arithmetic, there are infinitely many maximal lattices in $\mathcal{A}_{\Gamma}$.

In the joint paper with Rohlfs [86], a more general result is proved. Specifically, let $S$ be a finite set. For each $\nu$ in $S$, let $k_{\nu}$ be a nondiscrete locally compact field of characteristic zero and $\mathbf{G}_{\nu}$ be a semisimple algebraic group defined over $k_{\nu}$. Define

$$
G_{S}=\prod_{v \in S} \mathbf{G}_{v}\left(k_{\nu}\right)
$$

Then $G_{S}$ is a locally compact unimodular group and hence admits invariant measures. Let $\Gamma$ be a lattice in $G_{S}$. Define the set $\mathcal{A}_{\Gamma}$ of commensurable lattices $\Gamma^{\prime}$ in $G_{S}$ as above. It is shown in [86] that if $G_{S}$ has no compact factors, then there is an invariant measure $\omega$ on $G_{S}$ such that for every lattice $\Gamma^{\prime} \in \mathcal{A}_{\Gamma}$, the volume of $\Gamma^{\prime} \backslash G_{S}, \operatorname{vol}\left(\Gamma^{\prime} \backslash G_{S}\right)=\int_{G_{S} / \Gamma^{\prime}} \omega$, is an integer. Equivalently, for any invariant measure, the volumes of $\Gamma^{\prime} \backslash G_{S}, \Gamma^{\prime} \in \mathcal{A}_{\Gamma}$, are integral multiple of a common number.

\section{The Oppenheim CONJeCture on Values of QUADRATIC FORMS}

The Oppenheim conjecture on values of quadratic forms at integer points is the following statement:

Let $B$ be a nondegenerate real quadratic form in $n \geq 3$ variables. Assume that $B$ is indefinite and irrational, i.e., not a multiple of a quadratic form with rational coefficients. Then for every $\varepsilon>0$, there exist integers $x_{1}, \cdots, x_{n}$, not all equal to zero, such that $\left|B\left(x_{1}, \cdots, x_{n}\right)\right|<\varepsilon$.

Originally, the conjecture was made by Oppenheim in 1929 for $n \geq 5$, motivated by the following result of Mayer: If $B$ is a nondegenerate, indefinite real 
quadratic form in $n \geq 5$ variables and is a multiple of a quadratic form with rational coefficients, then $B$ represents 0 over $\mathbb{Z}$ nontrivially, i.e., there exist integers $x_{1}, \cdots, x_{n}$, not all equal to zero, such that $B\left(x_{1}, \cdots, x_{n}\right)=0$. This result does not hold for $n=3,4$. The above the Oppenheim conjecture was extended for $n \geq 3$ by Davenport. (See [97-3] for more detail on the history of this conjecture.)

Later in 1952, Oppenheim strengthened the conjecture to the following one: Under the same assumption as in the above conjecture, for every $\varepsilon>0$, there exist integers $x_{1}, \cdots, x_{n}$, not all equal to zero, such that

$$
0<\left|B\left(x_{1}, \cdots, x_{n}\right)\right|<\varepsilon .
$$

Oppenheim also proved that this stronger conjecture implies that the values $B\left(\mathbb{Z}^{n}\right)$ form a dense subset of $\mathbb{R}$.

Before this conjecture was completely proved by Margulis [87-1] [88-2] [89$3]$ [89-6], it was extensively studied by methods from analytic number theory, settling case-by-case mainly for quadratic forms in large number variables or special quadratic forms. The pace was slow and the approach using circle methods seems not sufficient to prove this conjecture for general quadratic forms in small number of variables.

Margulis used methods of dynamical systems to prove this conjecture in complete generality. The following recollection of Margulis explains well the history of his involvment with this conjecture and ideas of the proof:

"In 1978 I won a Fields Medal which was of course very encouraging. Unfortunately I was not able to come to the Helsinki Congress to receive the award because of the opposition of the top Soviet mathematical establishment (you can read more about that in the book by Lehto titled Mathematics without borders ${ }^{10}$ ). But apparently because I could not come to the Helsinki Congress I was allowed to come to the West for the first time in 1979. It was a 3 month visit to Bonn which was arranged by Hirzebruch. During that vist Jacques Tits came to Bonn and at a small ceremony presented the [Fields] award.

During my 1979 visit to Bonn I met Gopal Prasad who told me about the Raghunathan conjecture on closures of unipotent orbits. He also mentioned the remarkable observation by Raghunathan that this conjecture would imply the Oppenheim conjecture (actually at that time Raghunathan referred to the Oppenheim conjecture as the Davenport conjecture). This conversation with Gopal Prasad was quite inspiring and I started to think about the Oppenheim and Raghunathan conjectures. My earlier work on nondivergence to infinity of orbits of unipotent

\footnotetext{
${ }^{10}$ The precise information about this book is: Mathematics without borders: A history of the International Mathematical Union, Springer-Verlag, New York, 1998. xvi+399 pp.
} 
flows ${ }^{11}$ provided me with some intuition and enthusiasm (mostly because of the understanding of the importance of the polynomial divergence in the theory of unipotent flows). But it took quite a long time before I developed an approach to the proof of the Raghunathan conjecture based on the study of minimal sets and the behavior o orbits of unipotent flows near minimal sets. I eventually proved the Oppenheim conjecture in 1986 but some preliminary announcements were made before that. In particular, in August of 1984, I participated in a conference in Hungary. The conference was on a subject completely unrelated to homogeneous flows, but Katok, Kazhdan and Ratner were there. I gave a talk at the conference where I announced the proof of the Oppenheim conjecture and explained my approach towards the proof of the Raghunathan conjecture. ${ }^{12} 13$

In the late eighties it became much easier to travel to the West from the Soviet Union. In 1988 I spent 4 months at the Max Planck Institute in Bonn. There I met S.G.Dani and we started our collaboration which continued for several years. We proved first that the orbits of $S O(2,1)$ in $S L(3, \mathbb{R}) / S L(3, \mathbb{Z})$ are either closed or dense and after that proved the Raghunathan conjecture for generic unipotent subgroups in $S L(3, \mathbb{R})$. These results have interesting number theoretic applications, and the latter result was the first instance when the Raghunathan conjecture was proved for a non-horospherical subgroup of a semisimple Lie group. I am certain that the methods of [87-1], [89-2], [89-3], [89-4], [89-6] and [90-1] can be applied to prove the Raghunathan conjecture in many "small cases" such as the product of two copies of $S L(2, \mathbb{R})$ or, more generally, the product of two groups of the type $S L(2, K)$ where $K$ is either $\mathbb{R}$ or $\mathbb{Q}_{p}$ (it is worth mentioning that these methods were applied by Nimish Shah in his thesis to prove the Raghunathan conjecture for $S L(2, \mathbb{C}))$. It is apparently enough to consider such "small" cases for some applications. Actually, as I mentioned on several occsions, the just mentioned methods can be most probably applied to prove the Raghunathan conjecture in general (but in the general case there are many technical complications.) Of course, now it is not necessary to that because M.Ratner proved the Raghunathan conjecture by a different method.

\footnotetext{
${ }^{11}$ It is the result (ii) mentioned in the review of [69-3] by Garland quoted in $\S 7$.

${ }^{12}$ The Oppenheim conjecture certainly provided impetus to the theory of homogeneous flows and, in particular, to the approach of using homogenous flows to study number theoretic questions. See the next section and $\S 40$, and also the quote of Howe [Ho, p.262] at the beginning of this article.

${ }^{13}$ In [Bo3, p.14], Borel explained that "It is easily seen that it suffices to prove this conjecture for $\mathrm{n}=3$. Raghunathan's remark, also made around 1975, was that it [the Oppenheim conjecture] would follow from the following statement:

$(*)$ any relatively compact orbit of $S O(2,1)$ in $S L(3, \mathbb{R}) / S L(3, \mathbb{Z})$ is compact.

G.A.Margulis heard about it, took the hint, proved the statement about $S O(2,1)$ and hence the Oppenheim conjecture (see remark below) [89-3]. This breakthrough opened the way to an intense activity both on refinements of the Oppenheim conjecture, on the Raghunathan conjecture and a related one by Dani (see below)."
} 
After M.Ratner obtained around 1990 her fundamental results on unipotent flows, it became possible to study the quantitative version of the Oppenheim conjecture. First S.G.Dani and myself obtained lower bounds using the just mentioned result of Ratner and the so-called "linearization method" and later Eskin, Mozes and myself obtained upper bound and asymptotics. I should say that the main new ingredient in the joint work with Eskin and Mozes are certain integrability estimates. The proof of these estimates is based on some new methods. Some of these methods were later used in [04-2] and [04-4]. ${ }^{14}$ "

First, the Oppenhiem conjecture in more than three variables can be easily reduced to the case $n=3$. In this case, the Oppenheim conjecture is equivalent to a result on compactness of some orbits of orthogonal groups on the homogeneous space of unimodular lattices.

More specifically, let $B_{0}$ be an indefinite ternary quadratic form, and let $H=$ $H_{B_{0}}$ be the associated orthogonal group $S O\left(B_{0}\right)=\left\{g \in S L(3, \mathbb{R}) \mid g \cdot B_{0}=B_{0}\right\}$. For any point $z \in S L(3, \mathbb{R}) / S L(3, \mathbb{Z})$, denote its stabilizer in $H$ by $H_{z}$.

Then the following two statements are equivalent:

(1) The Oppenheim conjecture is true, i.e., for every irrational indefinite ternary quadratic form $B$, and for every $\varepsilon>0$, there exists a non-zero integral vector $v \in \mathbb{Z}^{3}$ such that $|B(v)|<\varepsilon$.

(2) If for a point $z \in S L(3, \mathbb{R}) / S L(3, \mathbb{Z})$, the orbit $H z$ is relatively compact in $S L(3, \mathbb{R}) / S L(3, \mathbb{Z})$ (i.e., its closure is compact), then it is compact, i.e., the quotient space $H / H_{z}$ is compact.

This relation between the Oppenheim conjecture and problems on dynamical systems on homogeneous spaces was observed by Raghunathan in mid-seventies and opened up a completely new way to attack the Oppenheim conjecture.

In fact, Raghunathan also made a more general conjecture on closures of unipotent group actions on homogeneous spaces: Let $G$ be a Lie group, $\Gamma$ be a lattice in $G$ and $\{u(t)\}, t \in \mathbb{R}$, be a Ad-unipotent one-parameter subgroup of $G$. Then for any $x \in G / \Gamma$, there exists a closed connected subgroup $F$ of $G$ such that the closure $\overline{\{u(t) x \mid t \in \mathbb{R}\}}=F x$, and the orbit $F x$ admits a finite $F$-invariant probability measure.

Closely related to the above conjecture of Raghunathan is a conjecture of Dani (see $[97-3,3.8]$ ): If $G$ is a connected Lie group, $\Gamma \subset G$ is a lattice, $U$ is an Adunipotent subgroup of $G$, and $\mu$ is a finite Borel $U$-invariant measure on $G / \Gamma$, then there exists a closed subgroup $F$ of $G$ such that $\mu$ is $F$-invariant and Supp

\footnotetext{
${ }^{14} \mathrm{~A}$ basic result in the reduction theory of arithmetic subgroups is that the covolume of an arithmetic subgroup of a semisimple Lie group is finite. This is reproved in these papers [04-2] and [04-4] by using random walks on homogeneous spaces.
} 
$(\mu)=F x$ for some $x \in G / \Gamma$. (In the above conjecture, a subgroup $U$ of $G$ is Ad-unipotent if the linear transformation $\operatorname{Id}-\operatorname{Ad}_{G} u$ is nilpotent for every $u \in U$.)

It should be stressed that the work of Dani on unipotent group actions has played a crucial role in the insight of Raghunathan in formulating the above conjectures. In fact, in an email message to Margulis in 1996, Raghunathan explained that his interest in orbit closures of subgroups was triggered by some early work of Dani (in collaboration with his wife J.S.Dani and S.Raghavan). He could interpret the main result of their paper as the density of an orbit in $G / \Gamma$ for a subgroup $\Gamma$ of $G$. Later Dani formally enrolled as a student for the Ph.D degree and was interested in studying flows on homogeneous spaces, and Raghunathan started learning with him some ergodic theory especially in the context of these homogeneous spaces. Various results of Dani and others on horosphere flows on homogeneous spaces led Raghunathan to formulate the above conjecture for the closure of one parameter Ad-unipotent subgroups and later to more general Ad-unipotent groups. Around the same time, Dani also formulated the stronger conjecture on classification of measures invariant under Ad-unipotent subgroups, which was finally proved by Ratner [Ra6] (See [Da6] and also [Da8], [97-3, §2.3, $\S 3.8]$ ). In the late sixties and early seventies, some of colleagues of Raghunathan were interested in the Oppenheim conjecture and he became interested in the question after a talk given by one of them. Raghunathan was at that time trying very hard to prove the result that unipotent 1-parameter groups can not have closed non-compact orbits and the proof of Margulis appeared. ${ }^{15}$ Raghunathan had realized that the Oppenheim conjecture was a statement about the noncompactness of an orbit under the orthogonal group and it was evident that the difference between dimension 2 and the higher ones was the presence of unipotents in the orthogonal group. Raghunathan's efforts at understanding Dani's theorems on horospheres and the proof of Margulis together with the above observation about the Oppenheim conjecture led him to speculate more boldly. It was his hope that one could use a downward induction on dimension starting with a horosphere. The slow growth of the unipotent 1-parameter group in contrast to the diagonal situation was at the back of his mind and made him hope that these flows are likely to have managable behaviours.

See [Da6], [Da4] [Da7] for more details and summaries of some work of Dani, and also [Bo3, pp. 13-15] for some complementary explanations of the history of the Oppenheim conjecture.

As Margulis pointed out in [97-3, §2.3], such a relation was implicitly contained and used in a paper of Cassels and Swinnerton-Dyer in 1955, though not in such languages of dynamical systems.

\footnotetext{
${ }^{15}$ See the result (ii) in the quote by Garland in $\S 7$.
} 
After the initial breakthrough by Margulis, there have also been various improvements, for example, the stronger version of the conjecture requiring the non-vanishing of the value $\left|B\left(x_{1}, \cdots, x_{n}\right)\right|$. As pointed out before, this implies that the values of $B$ on integral points form a dense subset of $\mathbb{R}$. In the joint papers with Dani [89-2] [89-4] [90-2], it was shown that the values of $B$ on primitive integral points also form a dense subset of $\mathbb{R}$. A generalization of this result was proved by Borel and Prasad in [BoP]. See [97-3] for a survey.

The earlier successes on the Raghunathan conjecture by Margulis also motivated a lot of work on the more general Raghunathan conjecture. The papers [90-1] [91-2] (joint with Dani) [90-3] are related to this conjecture. The papers [88-2] [91-4] [97-3] give summaries and surveys of these and related results, and [97-3] is probably the most comprehensive one. The article [00-3] contains many open problems.

The conjectures of Dani and Raghunathan are proved in full generality by Ratner. As Prasad pointed out in the review of Margulis' paper [89-6]:

"With the impetus provided by these papers of the author ([87-1], [89-3], [896]), there has recently been a very important development in the area: M. Ratner has proved Raghunathan's conjecture ...."

It should be pointed out that in [89-3] and [88-2], Margulis explicitly stated the conjectures of Raghunathan and Dani, and also gave some generalizations of these conjectures. Also in [88-2] Margulis, following S.G.Dani, stated the conjecture about the uniform distribution of orbits of unipotent flows.

Three of main theorems of Ratner are the following [Ra2-6]:

(1) (Classification of invariant measures). Let $U$ be a subgroup of $G$ generated by Ad-unipotent elements. If $\mu$ is any ergodic $U$-invariant probability measure on $G / \Gamma$, then there is a closed subgroup $H$ of $G$, such that the support of $\mu$ is a single $H$-orbit, and $\mu$ is $H$-invariant.

(2) (Classification of orbit closures). Let $U$ be a subgroup of $G$ generated by $A d$-unipotent elements. The closure of each $U$-orbit on $G / \Gamma$ is the support of some ergodic $U$-invariant probability measure $\mu$.

(3) (Uniform distribution). Let $U=\{u(t)\}$ be a one-parameter Ad-unipotent subgroup of $G$. For every $x \in G / \Gamma$, there is an ergodic $U$-invariant probability measure $\mu$ on $G / \Gamma$ such that the $U$-orbit $\{u(t) x\}$ is uniformly distributed with respect to $\mu$.

These results play an important role in the quantitative version of the Oppenheim conjecture to be discussed below and in many other applications, which include on uniform distribution of Heegner points [Vat], sharp bounds on various counting problems [Es1] [Es2], determination of the distribution of normalized 
gaps (or spacings) in the sequence of fractional parts of $\sqrt{n}, n \in \mathbb{N}[$ ElM] (see also [Ra1] and [Mor] [Da7] for more details).

The joint papers of Margulis with Tomanov [92-2] [94] give a much shorter proof of the crucial theorem on classification of invariant measures of Ratner in the case where $G$ is algebraic. These papers also give the generalization of this result to the case where $\Gamma$ are discrete subgroups and $\mathrm{G}$ are products of real and $p$-adic algebraic Lie groups, which was also proved in a more general context by Ratner in [Ra2]. The joint paper with Tomanov [96-2] gives a further generalization of this result by eliminating the assumption that $\Gamma$ is discrete.

\section{Quantitative version of the Oppenheim conjecture}

The Oppenheim conjecture formulated above is qualitative. A significant improvement on the solution of the Oppenheim conjecture is to give asymptotics of integral points $v \in \mathbb{Z}^{n}$ with small values $B(v)$ and to prove a quantitative version of the Oppenheim conjecture. Specifically, let $B$ be a real irrational indefinite quadratic form in $n$ variables with $n \geq 3$. For every open interval $(a, b) \subset \mathbb{R}$, and $T>0$, define the counting function

$$
N_{(a, b)}^{B}(T)=\left\{v \mid v \in \mathbb{Z}^{n}, \text { with }|v|<T, a<B(v)<b\right\} .
$$

By the positive solution of the the stronger version of the Oppenheim conjecture, which requires $\left|B\left(x_{1}, \cdots, x_{n}\right)\right|>0$ and implies that the values $B(v), v \in \mathbb{Z}^{n}$, are dense in $\mathbb{R}$, it follows that when $T \rightarrow+\infty$,

$$
N_{(a, b)}^{B}(T) \rightarrow+\infty .
$$

Since the number of integral points in a large ball in $\mathbb{R}^{n}$ is proportional to its volume, it is natural to expect that $N_{(a, b)}^{B}(T)$ is asymptotic to a similarly defined volume, i.e., as $T \rightarrow+\infty$,

$$
N_{(a, b)}^{B}(T) \sim \operatorname{vol}\left(\left\{v \in \mathbb{R}^{n}|a<B(v)<b,| v \mid<T\right\}\right) \sim \lambda(b-a) T^{n-2},
$$

where $\lambda=\lambda(B)$ is a positive constant depending on $B$. This result turns out to be true if the signature $(p, q)$ of the quadratic form $B$ satisfies $p \geq 3$ and $q \geq 1$.

The joint paper with Dani [93] gives lower bounds on $N_{(a, b)}^{B}(T)$ without any restriction on the signature of $B$. For this purpose, they proved a strengthened version, i.e., a uniform version, of the theorem of Ratner on equidistribution of individual orbits of unipotent flows. This result was proved by a linearization procedure.

The upper bound on and the asymptotics of $N_{(a, b)}^{B}(T)$ as $T \rightarrow+\infty$ are much more difficult and achieved in the joint papers with Eskin and Mozes [95-3] [981] [05-1]. In fact, the method from [93] can not be applied, and without the restriction on the signature mentioned above, i.e., $p \geq 3$ and $q \geq 1$, the sharp 
upper bound on $N_{(a, b)}^{B}(T)$ as stated above does not hold. Briefly, as noticed by P.Sarnak, for a quadratic form $B_{\alpha}(x)=x_{1}^{2}+x_{2}^{2}-\alpha^{2} x_{3}^{2}$, if $\alpha$ is too rapidly approximated by rational numbers, then the number $N_{(a, b)}^{B}(T)$ will grow faster than $T^{n-2}=T$, i.e., $N_{(a, b)}^{B}(T) / T$ is not bounded as $T \rightarrow \infty$. The paper [98-1] proves that the same conclusion holds for quadratic forms

$$
B_{\alpha}(x)=x_{1}^{2}+x_{2}^{2}-\alpha^{2}\left(x_{3}^{2}+x_{4}^{2}\right) .
$$

Specifically, for every $\varepsilon>0$, there exist an irrational form $B$ of signature $(2, q)$ with $q=1,2$, a sequence $T_{j} \rightarrow \infty$, and a constant $c>0$ such that

$$
N_{(a, b)}^{B}\left(T_{j}\right)>c T_{j}^{q}\left(\log T_{j}\right)^{1-\varepsilon} .
$$

On the positive side, [98-1] proves that for any form $B$ of signature $(2, q)$ with $q=1,2$, there exists a constant $c>0$ such that

$$
N_{(a, b)}^{B}(T)<c T^{q} \log T, \quad T>2 .
$$

Moreover, for almost all such forms $B$, i.e. of signature $(2,1)$ or $(2,2)$, the following asymptotic formula holds:

$$
N_{(a, b)}^{B}(T) \sim \lambda(b-a) T^{q} .
$$

A crucial step in the proof of the upper bounds on and hence the asymptotics of $N_{(a, b)}^{B}(T)$ in [98-1] is to get precise control on the behaviors of orbits of semisimple subgroups on the space of lattices $S L(n, \mathbb{R}) / S L(n, \mathbb{Z})$.

For quadratic forms of signature $(2,2)$ satisfying certain Diophantine conditions, the above asymptotics on $N_{(a, b)}^{B}(T)$ were obtained in [05-1]. Briefly, a quadratic form $B$ is called extremely well approximable by split forms (EWAS) if for any $N>0$ there exists a split integral form $B^{\prime}$ and $k \geq 2$ so that $\left\|B-\frac{1}{k} B^{\prime}\right\| \leq 1 / k^{N}$. For example, if $\beta$ is a Liouville number, i.e., rapidly approximated by rational numbers, then the form $B_{\beta}=q_{\beta}\left(x_{1}, x_{2}\right)-q_{\beta}\left(x_{3}, x_{4}\right)$, where $q_{\beta}(x, y)=x^{2}+\beta y^{2}$, is EWAS. As pointed out above, for such form, $N_{(a, b)}^{B}(T)$ may grow faster than $T^{2}=T^{n-2}$.

Another source for violating the desired upper bound comes from integral points on rational isotropic subspaces with respect to the quadratic form $B$.

Let $\tilde{N}_{(a, b)}^{B}(T)$ be the number of integral points $v$ which do not lie on the rational isotropic subspaces and satisfy the bounds: $|v|<T, a<B(v)<b$. Then one of the main results in [05-1] is that if a quadratic form $B$ of signature $(2,2)$ is not EWAS, then $\tilde{N}_{(a, b)}^{B}(T)$ satisfies the desired asymptotics above:

$$
\tilde{N}_{(a, b)}^{B}(T) \sim \operatorname{vol}\left(\left\{v \in \mathbb{R}^{n}|a<B(v)<b,| v \mid<T\right\}\right) \sim \lambda(b-a) T^{n-2} .
$$

An important consequence of this result is an explicit construction of many flat tori whose pair correlation function for the eigenvalues of the Laplacian satisfies 
the Berry-Tabor conjecture. Sarnak showed a generic flat torus satisfies the Berry-Tabor conjecture for the pair correlation function, but the method does not produce any explicit example. Indeed, no explicit example was known before [05-1].

The methods developed in [98-1] for the sharp upper bound on $N_{(a, b)}^{B}(T)$ have also be effectively used in other problems, for example, in the problem of asymptotics for the number of cylinders and saddle connections on flat surfaces [EsM], counting problems for billards [EsMS], and for obtaining quantitative recurrence and large deviations results for the Teichmuller geodesic flow on connected components of strata of the moduli space of holomorphic unit-area quadratic differentials on compact surfaces [At]. See also [EMS] for related counting lattice points on homogeneous varieties and [Es2] for an introduction to counting problems in moduli space.

\section{Oseledets multiplicative ERGODiC TheOREM AND LyapunOV EXPONENTS}

The joint paper with Goldsheid [87-2] gives conditions under which the Lyapunov exponents of linear multidimensional stochastic difference equations are distinct. Briefly, let $\left\{\xi_{n}\right\}$ be a stationary Markov chain with state space $S$, invariant distribution $\pi(\cdot)$, and transition kernel $P(\cdot, \cdot)$. Suppose that $f: S \rightarrow G L(d, \mathbb{R})$ is a Borel measurable function. Then the Oseledets multiplicative ergodic theorem applies to the stationary matrix sequence $\left\{f\left(\xi_{n}\right)\right\}_{n=1}^{\infty}$ and gives an increasing sequence $\gamma_{1} \leq \cdots \leq \gamma_{d}$ of Lyapunov exponents and a filtration of random subspaces $V_{i}$ of $\mathbb{R}^{d}$, which are defined by

$$
V_{i}=\left\{v \in \mathbb{R}^{d}: \lim n^{-1} \log \left\|f\left(\xi_{n}\right) f\left(\xi_{n-1}\right) \cdots f\left(\xi_{1}\right) v\right\| \leq \gamma_{i}\right\} .
$$

One problem is to obtain conditions under which the Lyapunov exponents $\gamma_{i}$ are all distinct and the dimension of $V_{i}$ is equal to $i$.

The joint paper with Goldsheid [89-5] proves the following important theorem: Let $A_{1}, A_{2}, \cdots$ be independent random matrices in $G L(d, \mathbb{R})$ with the same law $\mu$. Let $H$ be the semigroup generated by the support of $\mu$ and $H^{\prime}$ be the algebraic closure of $H$. If $H^{\prime}=G L(d, \mathbb{R})$, then all the Lyapunov exponents of the sequence $\left\{A_{n} A_{n-1} \cdots A_{1}, n \geq 1\right\}$ are distinct. Similarly, a criterion ensuring that the leading exponent is simple is given. It depends only on $H^{\prime}$. This paper also gives a simplified proof of Oseledets multiplicative ergodic theorem with extension to the infinite-dimensional case. This Oseledets multiplicative ergodic theorem plays an important role in the super-rigidity of irreducible lattices of higher rank semisimple Lie groups, in particular, in proving that the Zariski closure of the image $\pi(\Gamma)$ of the lattice $\Gamma$ (see $\S 9$ ) is necessarily reductive. 
The joint paper with Karlsson [99-2] generalizes the Oseledets multiplicative ergodic theorem as follows: Let $(X, \mu)$ be a probability space, $T: X \rightarrow X$ be an ergodic measure preserving transformation. Let $(Y, d)$ be a nonpositively curved space (or a CAT(0)-space), $D \subset Y$ a nonempty subset, $y \in D, S$ the set of nonexpanding maps $D \rightarrow D$, and $\varphi: X \rightarrow S$ such that $\int_{X} d(y, \varphi(x) y) d \mu(x)<\infty$. For $x \in X$ and $n \in \mathbb{N}_{0}$, let $y_{n}(x)=\varphi(x) \varphi(T(x)) \cdots \varphi\left(T^{n-1}(x)\right) y$. Then for almost all $x \in X$, the $\operatorname{limit}_{n \rightarrow \infty} \lim _{n \rightarrow \infty} d\left(y, y_{n}(x)\right) / n$ exists, which is denoted by $A$; and if $A>0$, then for almost every $x$, there is a unique geodesic ray $\gamma_{x}$ in $Y$ starting at $y$ such that $\lim _{n \rightarrow \infty} d\left(\gamma_{A n}(x), y_{n}(x)\right)=0$.

The usual multiplicative ergodic theorem is obtained by taking $Y=\operatorname{GL}(N, \mathbb{R}) /$ $\mathrm{O}(N, \mathbb{R})$ and hence $S=\mathrm{GL}(N, \mathbb{R})$. As an application, the paper gives a characterization of the Poisson boundary with respect to the probability measure of a countable group of isometries of a complete uniformly convex nonpositively curved metric space.

\section{Proximal Linear maps}

An invertible, real matrix is called proximal if it has a unique eigenvalue of maximum absolute value. The study of such matrices is useful for several applications. One reason is that a proximal matrix induces a map on the projective space with a unique attracting fixed point whose basin is equal to a complement of a hyperplane. This fact plays an important role in the proof of Tits alternative for linear groups.

Proximal elements in any semisimple Lie group $G$ are also used by Furstenberg to study the maximal Furstenberg boundary $B(G)$ of $G$, which are crucial for many applications in the super-rigidity of lattices, boundary values and integral representations of harmonic functions on the symmetric space associated with $G$. In fact, he used the existence of proximal elements in $G$ to show that $B(G)$ is determined measure-theoretically by any lattice in $G$, and used it to prove a special case of Mostow-Margulis strong rigidity: a lattice in $\operatorname{SL}(2, \mathbb{R})$ is not isomorphic to a lattice in $\operatorname{SL}(n, \mathbb{R})$ for $n>2$.

In the joint paper with Goldsheid [89-5], it was proved that if a connected Lie subgroup $G \subset \mathrm{GL}(V)$ contains a proximal element, then so does every Zariskidense subsemigroup $H$ of $G$, provided $V$ is irreducible as a representation of $\bar{G}^{0}$, the connected component of the Zariski-closure of $G$. One of the main results of the joint paper with Abels and Soifer [95-2] is that, under the same conditions, there is a subset $M$ of $H$ with $\operatorname{dim}(V)^{2}$ elements such that, for any $g$ in $G$, there exists some element $m \in M$ such that $m g$ is proximal. A quantitative version is also obtained, and hence there is a rich supply of proximal elements in $G$. This paper also gives a criterion for the image of a reductive group under an irreducible representation to contain a proximal element. This criterion is given in terms of 
the highest weight of the representation and implies that when $G$ is $\mathbb{R}$-split, there are always proximal elements.

\section{QC-MAPS AND CARNot-CaRAthÉOdory SPACES}

Quasiconformal (abbreviated qc) maps are basic objects in complex analysis of one variable, for example, in the theory of Teichmuller spaces of Riemann surfaces. The notion of qc maps makes sense for general metric spaces. In fact, for every $K \geq 1$, a $K$-qc map between any two metric spaces $X_{1}, X_{2}$ is a homeomorphism $f: X_{1} \rightarrow X_{2}$ such that the infinitesimal distortion from a circle is bounded by $K$, i.e.,

$$
\limsup _{r \rightarrow 0} L_{r}(p) / l_{r}(p) \leq K
$$

for all $p$, where $L_{r}(p)$ [resp. $\left.l_{r}(p)\right]$ denotes the sup [resp. the inf] of $d(f(p), f(q))$ as $q$ varies over the sphere $d(p, q)=r$.

But they have only recently been studied in such a general context. In fact, before Mostow used qc maps in the context of the non-isotropic metrics of the boundaries of rank one symmetric spaces of noncompact type to prove his wellknown strong rigidity in [Mos1] (see also the earlier paper [Mos2]), qc maps were only studied on $\mathbb{R}^{n}$ with respect to the standard metric.

After this, the theory of qc maps have been developed for other groups, for example, Heisenberg groups by Koranyi and Riemann [KoR1-2], and general nilpotent groups by Pansu [Pan], Heinonen and Holopainen [HeH], and Vodopyanov [Vo].

The joint paper with Mostow [95-1] studies what seems to be a natural and broad context to which the classical theory of qc maps can be generalized. The group structure is dropped and the question is studied on manifolds equipped with a finite Carnot-Carathéodory distance generated by a distinguished "horizontal" subbundle of the tangent bundle with a norm defined on it. This generalization corresponds exactly to the classical generalization of the $\mathbb{R}^{n}$-theory to Riemannian manifolds and to the generalization of the Heisenberg group theory to strongly pseudoconvex CR manifolds. Three fundamental results of the theory are generalized to the present context: The older result of Mostow on absolute continuity on lines, the result of Koranyi and Riemann on the Heisenberg groups, that the inverse of a qc map is qc, and Pansu's theorem on a.e. differentiability which is now formulated in terms of the Gromov tangent cone to the manifold.

The main purpose of the joint paper with Mostow [00-4] is to give an improved definition of the "tangent cone" of a manifold with Carnot-Carathéodory metric. This notion was introduced by Gromov and was later studied [95-1], but it was not uniquely defined. 


\section{BoundeD ORBITS AND UNBOUNDED ORBITS OF FLOWS}

It is a natural and important problem to understand structures of orbits of dynamical systems. There are two types of orbits: bounded and unbounded.

Let $G$ be a Lie group, $\Gamma \subset G$ be a lattice subgroup, and $F \subset G$ be a oneparameter subgroup. The joint paper with Kleinbock [96-1] describes the set of bounded $F$-orbits on the homogeneous space $G / \Gamma$.

If $G / \Gamma$ is non-compact and $F$ is unipotent and induces an ergodic flow, then it follows from a result of Ratner [Ra3] that the set of bounded $F$-orbits lies in a countable union of proper submanifolds of $G / \Gamma$, and hence in this case the set of bounded orbits is of Hausdorff dimension at most $\operatorname{dim}(G)-1$. On the other hand, if $G=\operatorname{SL}(n, \mathbb{R}), \Gamma=\operatorname{SL}(n, \mathbb{Z})$, and $F$ is a diagonal subgroup of $G$ with two eigenvalues, then Dani [Da5] proved that the set of bounded $F$-orbits is of full Hausdorff dimension.

This leads to the following question: Under what conditions is the set of bounded orbits large enough? The paper [96-1] completely settles this problem, after an earlier related work of Dani [Da3] for the rank one case under an assumption that the adjoint action of the one parameter group has an eigenvalue of absolute value other than 1 . The main case to study is when $\Gamma$ is an irreducible lattice in a semisimple Lie group $G$. The paper [96-1] proves that for any partially hyperbolic (i.e. nonquasiunipotent) one parameter subgroup $F$, the set of bounded $F$-orbits on $G / \Gamma$ is of full Hausdorff dimension.

Instead of bounded orbits, unbounded orbits and their asymptotic behaviors are considered in the joint paper with Kleinbock [99-1]. Let $G$ be a semisimple Lie group and $\Gamma$ a lattice in $G$ as above. Let $\mu$ be the $G$-invariant probability measure on $G / \Gamma$. Let $\left\{g_{i}\right\}$ be a sequence of elements in $G$ and $\left\{E_{i}\right\}$ be a sequence of subsets of $G / \Gamma$. The general problem in [99-1] is to understand conditions under which $g_{i} x \in E_{i}$ for infinitely many $i$, for $\mu$-almost all $x$ in $G / \Gamma$. This paper describes a condition on $\left\{g_{i}\right\}$, called exponential divergence, and a class of functions called distance-like functions, such that if $\Delta$ is a distance-like function and $E_{i}=\left\{x \in G / \Gamma \mid \Delta(x) \geq t_{i}\right\}$ for a sequence $\left\{t_{i}\right\}$ such that the series $\sum_{i} \mu\left(E_{i}\right)$ is divergent, then the desired conclusion holds.

When specialized to $G=S O(n, 1)$, this result gives a generalization to higher rank and a sharpening of Sullivan's logarithm law for geodesics of hyperbolic manifolds [Sul2]. It also gives a new proof of the classical theorem of Khinchin and Groshev on simultaneous Diophantine approximation.

These and other related results are also discussed in [00-3] [02-3]. 


\section{Compact quotients of homogeneous SPaCeS}

If $G$ is a noncompact semisimple Lie group with finite center, and $H$ is a compact subgroup of $G$, then a result of Borel implies that there always exists discrete subgroups $\Gamma$ that acts properly on $G / H$ with compact quotient.

On the other hand, if $H$ is noncompact, the answer is not always true. In fact, in this case, no co-compact lattice $\Gamma$ can act properly on $G / H$.

One problem is to find conditions or obstructions to existence of such discrete groups. There have been some obstructions constructed before. The paper [97-2] gives another obstruction by studying the restriction to $H$ of matrix coefficients of unitary representations of $G$. Briefly, the obstruction says: Suppose that $H$ is not compact and there exists a compact subgroup $K \subset G$ and an integrable function $q$ on $H$ such that for any unitary representation $\pi$ of $G$ without nontrivial $G$-invariant vectors, any $K$-invariant vectors $w_{1}$ and $w_{2}$, and any $h \in H$, the following inequality holds:

$$
\left|\left\langle\pi(h) w_{1}, w_{2}\right\rangle\right| \leq q(h) \mid w_{1}\|\| w_{2} \| .
$$

Then $G / H$ has no compact quotients by discrete subgroups.

Therefore getting good bounds on matrix coefficients is important for this problem. Combined with some estimates by $\mathrm{Oh}[\mathrm{Oh} 1]$ on the matrix coefficients, this criterion gives many new examples of homogeneous spaces $G / H$ which do not admit proper and co-compact discrete group $\Gamma$-actions. Other sharp bounds on matrix coefficients and applications to computing explicitly the Kazhdan constant are obtained in [Oh2]. For other related results, see also [OhW].

The joint paper with Goldman [00-5] gives a proof of an unpublished result of Mess: there is no faithful, proper, isometric action of the fundamental group of a closed hyperbolic surface on $(2+1)$-dimensional Minkowski space $\mathbb{R}_{1}^{3}$. This contrasts with the existence result of many such actions if the hyperbolic surface is not assumed to be closed.

This problem on such proper and cocompact actions of discrete subgroups on non-Riemannian homogeneous spaces is still not fully understood. See the survey article for $[\mathrm{KoY}]$ the current status and many references.

\section{Metric Diophantine approximation and Khintchine-type THEOREMS}

The joint paper with Kleinbock [98-2] presents a new approach to metric Diophantine approximation on manifolds based on the correspondence between approximation properties of numbers and orbit properties of certain flows on homogeneous spaces. It gives a new proof of a conjecture of Mahler in the 1930s and 
settles conjectures of Baker and Sprindzhuk formulated in the 1970s. An important point here is that the number theoretic results were derived from estimates for flows on the space of lattices as mentioned in $\S 15$.

For $\mathbf{x} \in \mathbb{R}^{n}$, let

$$
\|\mathbf{x}\|=\max _{1 \leq i \leq n}\left|x_{i}\right|, \quad \Pi(\mathbf{x})=\prod_{i=1}^{n}\left|x_{i}\right| .
$$

A vector $\mathbf{x} \in \mathbb{R}^{n}$ is said to be very well approximated (VWA) if for some $\varepsilon>0$, there are infinitely many $q \in \mathbb{Z}$ and $\mathbf{p} \in \mathbb{Z}^{n}$ such that

$$
\|q \mathbf{x}+\mathbf{p}\|^{n}|q| \leq|q|^{-\varepsilon} \text {. }
$$

Similarly, a vector $\mathbf{x} \in \mathbb{R}^{n}$ is defined to be very well multiplicatively approximated (VWMA) if for some $\varepsilon>0$, there are infinitely many $q \in \mathbb{Z}$ and $\mathbf{p} \in \mathbb{Z}^{n}$ such that

$$
\Pi(q \mathbf{x}+\mathbf{p})|q| \leq|q|^{-\varepsilon} .
$$

Clearly, a vector is VWMA if it is VWA. It is also easy to show that almost every $\mathbf{x} \in \mathbb{R}^{n}$ is not VWMA. On the other hand, it is more difficult if points $\mathbf{x}$ are restricted to a proper submanifold $M \subset \mathbb{R}^{n}$. In the $1930 \mathrm{~s}$, Mahler conjectured that almost all points on the curve

$$
M_{0}=\left\{\left(t, t^{2}, \cdots, t^{n}\right) \mid t \in \mathbb{R}\right\} \subset \mathbb{R}^{n}
$$

are not VWA. This was settled by Sprindzhuk (see the survey article [Spr1] and the book [Spr2] for summaries and references).

Later in late 1970s, Sprindzhuk made the following conjecture: Let $f_{1}, \cdots, f_{n}$ be real-analytic functions in $\mathbf{x} \in U$, where $U$ is a domain in $\mathbb{R}^{d}$, which together with 1 are linearly independent functions over $\mathbb{R}$. Then almost all points of $M=$ $\left\{\left(f_{1}(x), \cdots, f_{n}(x)\right) \mid x \in U\right\}$ are not $V W A$.

The case $n=2$ of the conjecture was settled by Schmidt [Sch] in 1964, and the case $n=3$ was settled in 1996 by Beresnevich and Bernik [BeB].

A stronger conjecture (also formulated by Sprindzhuk) states that almost all points of $M$ are not VWMA. When $M=M_{0}$ defined above, this conjecture is known as Baker's conjecture. Except for the case $n=2$, the Baker conjecture has not been proved before.

The paper [98-2] proves a very general result which settles both of the conjectures of Sprindzhuk and Baker.

In the joint paper with Bernik and Kleinbock [01-2], the convergence case of a Khinchin type theorem is proved for non-degenerate manifolds. The result improves and extends previous work of Baker, Sprindzhuk, Bernik and others who had similar results for specific manifolds or for more restricted classes of manifolds. 
A point $x$ in $\mathbb{R}^{n}$ is called $\psi$-approximable if there exist infinitely many vectors $q$ in $\mathbb{Z}^{n}$ such that $|\langle x \cdot q\rangle| \leq \psi\left(|q|^{n}\right)$. Here, $|\cdot|$ denotes the supremum norm and $|\langle\cdot\rangle|$ denotes the distance from the integers. A point $x$ in $\mathbb{R}^{n}$ is called $\psi$-multiplicatively approximable if there exist infinitely many vectors $q$ in $\mathbb{Z}^{n}$ such that

$$
|\langle x \cdot q\rangle| \leq \psi\left(\prod_{+}(q)\right),
$$

where $\prod_{+}(q)=\prod_{i=1}^{n} \max \left(\left|q_{i}\right|, 1\right)$, the product of the non-zero entries of $q$.

Let $f=\left(f_{1}, \ldots, f_{n}\right)$ be an $n$-tuple of $C^{m}$ functions going from $U$ to $\mathbb{R}$, where $U$ is an open subset of $\mathbb{R}^{d}$. Then $f$ is nondegenerate at a point if the space $\mathbb{R}^{n}$ can be spanned by partial derivatives, up to some order, of $f$ at the point. Similarly the notion of non-degeneracy can be defined for the $d$-dimensional submanifold $M \subset \mathbb{R}^{n}$.

A manifold $M$ is said to be of Groshev type for divergence (resp. convergence) if almost all (resp. almost no) points of $M$ are $\psi$-approximable when the sum $\sum_{n=1}^{\infty} \psi(n)$ diverges (resp. converges).

The joint paper with Bernik and Kleinbock [01-2] proves that non-degenerate manifolds are of Groshev-type for convergence, and the joint paper with Beresnevich, Bernik and Kleinbock [02-2] proves that all nondegenerate manifolds are of Groshev type for divergence.

The article [02-3] surveys these and related results.

\section{Hyperbolic Penrose tile}

The Penrose tiles $[\mathrm{Pe}]$ comprise an aperiodic tiling system for $\mathbb{R}^{2}$. The Penrose tiles are not convex. Ammann has given an aperiodic tiling system consisting of three convex polygons (see [AmGS] and [Sen]). The existence of an aperiodic tiling system for $\mathbb{R}^{2}$ consisting of a single tile is still an open problem, although Penrose has given an example of such a system in the hyperbolic plane $\mathbb{H}^{2}$, but the tile is not convex. The joint paper with Mozes [98-3] constructs for each $n \geq 3$ a convex $n$-gon in $\mathbb{H}^{2}$ that tiles $\mathbb{H}^{2}$ but never periodically, i.e., the tile does not factors through a compact quotient of $\mathbb{H}^{2}$ by a discrete group of isometries acting freely on $\mathbb{H}^{2}$.

For aperiodic tilings of symmetric spaces and related results, see papers [Moz2] and $[\mathrm{BlW}]$.

\section{WiENER ERGODICITY FOR SEMISIMPLE LIE GROUPS}

Given a measure-preserving action $T_{v}: X \rightarrow X, v \in \mathbb{R}^{d}$, of the group $G=\mathbb{R}^{d}$ on a probability space $(X, m)$, and a function $f \in L^{1}(X)$, consider the averaging 
operators

$$
\pi\left(\beta_{t}\right) f(x)=\frac{1}{\operatorname{vol}\left(B_{t}\right)} \int_{v \in B_{t}} f\left(T_{v} x\right) d v
$$

where $B_{t}=\left\{v \in \mathbb{R}^{d}|| v \mid \leq t\right\}$.

Wiener's pointwise ergodic theorem asserts that $\pi\left(\beta_{t}\right) f(x)$ converges to a limit as $t \rightarrow \infty$ for almost every $x \in X$. The limit is given by the average of $f$ on $X$, $\int_{X} f d m$, provided the action is ergodic.

The joint paper with Nevo and Stein [00-2] considers the following generalization by replacing $\mathbb{R}^{n}$ by a connected semisimple Lie group with finite center and without compact factor. Let $K \subset G$ be a maximal compact subgroup, and $X=G / K$ be given a $G$-invariant Riemannian metric. For any ergodic measure-preserving action of $G$ on a probability space $(X, m)$, the ball averaging operators $\pi\left(\beta_{t}\right)$ can be defined similarly. The analogue of the Wiener ergodic theorem asserts that the averaging operators $\pi\left(\beta_{t}\right) f(x)$ converge to the average value $\int_{X} f d m$, for all $f \in L^{1}(X)$, or at least for all $f \in L^{p}(X), p>1$.

One of the main results of this paper is to give a positive answer for $p>1$. The related strong maximal inequality in $L^{p}$ is also proved and used to establish this ergodicity result. Before this paper, this Wiener ergodicity was only known for rank 1 simple Lie groups.

\section{Finite COVERINGS OF HyPERBolic MANifoldS}

A well-known conjecture of Thurston asserts that every $n$-dimensional hyperbolic manifold of finite volume has a finite sheeted cover with a positive first Betti number. This was motivated by an important step for the Thurston geometrization program for three dimensional manifolds. Briefly, for an irreducible 3-dimensional manifold with infinite fundamental group, the non-vanishing of the first homology group is related to the existence of an immersed incompressible surface in it, which implies that the manifold is Haken and hence can be understood better (see [DuT]).

The above conjecture is equivalent to that every lattice subgroup in $\mathrm{SO}(n, 1)$ has a finite index subgroup which is mapped onto the infinite cyclic group. In [Lu2], Lubotzky showed a stronger result for some lattices $\Gamma$. It was shown there that for some arithmetic and non-arithmetic lattices one can even find a finite index subgroup which is mapped onto a non-abelian free group.

The joint paper with Vinberg [00-1] generalizes Lubotzky's method and result to a much larger family of groups. In particular, it shows that Coxeter groups which are not virtually abelian have this property. It also shows that many discrete subgroups of $S O(n, 1)$, which are not necessarily lattices, are virtually mapped onto non-abelian free groups. 


\section{A generalized Tits alternative}

The paper [00-6] is related to the class of results which go under the name of Tits alternative. First we recall that the famous Tits alternative states that a finitely generated linear group either contains a nonabelian free group or is virtually solvable, i.e., it contains a solvable subgroup of finite index [Ti2]. It has many important applications, for example, it is an important ingredient in Gromov's proof that finitely generated groups with polynomial growth are nilpotent, and it is also used to prove that a famous conjecture of von Neumann that non-amenable groups must contain free groups is true for linear groups.

A different proof of the Tits alternative using the Oseledets multiplicative ergodic theorem was later given by Guivarch $[\mathrm{Gu}]$.

Such an alternative has also been proved for other classes of groups, for example, for the mapping class groups of surfaces by McCarthy [Mc] and independently by Ivanov [Iv], for the outer automorphism group of free groups by Bestvina, Feighn and Handel [BFH1-2], for the polynomial automorphisms of $\mathbb{C}^{2}$, for the generalized triangle groups by Fine, Roehl, and Rosenberger, for generalized tetrahedron groups by Howie and Kopteva.

On the other hand, Ghys and Sergiescu [GhS] showed that the Tits alternative does not hold for subgroups of the group Homeo $\left(S^{1}\right)$ of homeomorphisms of the circle and even for subgroups of the group of smooth diffeomorphisms of $S^{1}$. In 1998, Ghys conjectured a possible replacement of the Tits alternative for subgroups of Homeo $\left(S^{1}\right)$. The main result of the paper [00-6] is a proof of this conjecture, i.e., it proves that if a group $G$ acts by homeomorphisms on $S^{1}$, then, either there is a $G$-invariant probability measure on $S^{1}$, or $G$ contains a free nonabelian subgroup.

\section{Rigidity of ACtions of Lie GRoups AND LATtices}

As a non-linear generalization of the Mostow strong rigidity and Margulis super-rigidity of irreducible lattices in higher rank semisimple Lie groups, the Zimmer program [Zi1] [Zi2] tries to understand and to classify actions of such Lie groups and their lattices on compact manifolds preserving various geometric structures. Such actions can be viewed either as a nonlinear finite-dimensional theory or as the study of homomorphisms into the infinite-dimensional group of diffeomorphisms of the compact manifold under discussion, and they are expected to enjoy Mostow and Margulis type rigidity properties.

One of the central questions in this program about the actions of higher real rank groups and higher rank lattices is whether or not they are all (or under some mild hypotheses) of an algebraic nature. It is conjectured that such actions are rigid. For example, one conjecture states that for ergodic actions of higher rank 
lattices $\Gamma$ on closed manifolds preserving the Lebesgue measure, the actions are of algebraic nature on an open dense set with the following building blocks:

(1) actions preserving a Riemannian metric;

(2) actions on nilmanifolds $N / \Lambda$ by automorphisms via a homomorphism $\Gamma \rightarrow$ $\operatorname{Aut}(N / \Lambda)$, where $N$ is a simply connected nilpotent Lie group, $\Lambda$ is a cocompact lattice in $N$ and $A u t(N / \Lambda)$ consists of all automorphisms of $N$ preserving $\Lambda$;

(3) actions on $H / \Lambda$ by left translation via a homomorphism $\Gamma \rightarrow H$, where $H$ is a connected Lie group and $\Lambda \subset H$ is a cocompact lattice.

These are called standard actions. It is also conjectured that the standard actions are locally rigid, i.e., any small perturbations of these actions are conjugations by diffeomorphisms.

Under some weak hyperbolicity conditions, the joint paper with Qian [01-1] proves $C^{0}$ - and $C^{\infty}$-local rigidity theorems for two classes of standard algebraic actions: (1) left translation actions of higher real rank semisimple Lie groups and their lattices on quotients of Lie groups by uniform lattices; (2) higher rank lattice actions on nilmanifolds by affine diffeomorphisms; (3) $C^{0}$-global rigidity of volume preserving, higher rank lattice Anosov actions on nilmanifolds with a finite orbit.

The proofs depend on local rigidity of some constant cocycles and Zimmer's cocycle super-rigidity theorem, which is a generalization of the Margulis superrigidity of lattices to this non-linear group situation.

The joint paper with Fisher [03] studies perturbations of constant cocycles for actions of higher rank semi-simple algebraic groups and their lattices. For ergodic actions, Zimmer's cocycle superrigidity theorem implies that the perturbed cocycle is measurably conjugate to a constant cocycle modulo a compact valued cocycle. The main point of this article is to see that a cocycle which is a continuous perturbation of a constant cocycle is actually continuously conjugate back to the original constant cocycle modulo a cocycle that is continuous and 'small'. Applications include local rigidity of affine and quasi-affine actions of higher rank semisimple Lie groups and their lattices. This paper also improves and extends Zimmer's cocycle superrigidity.

The joint paper with Fisher [05-3] proves local rigidity of actions of groups having property $(\mathrm{T})$ of Kazhdan. Specifically, let $\Gamma$ be a countable group having property $(\mathrm{T})$, and $M$ a smooth compact Riemannian manifold, $k \in \mathbb{N}$. Assume that $\Gamma$ acts on $M$ by $C^{k}$-isometries, and denote this action by $\rho$. For any $\varepsilon>0$, if a $C^{k}$-diffeomorphism action of $\Gamma$ on $M$ is close in the $C^{k}$-topology to the isometric action $\rho$, then it is conjugate to $\rho$ by a small $C^{k-\varepsilon}$-diffeomorphism. The same result holds for $C^{\infty}$-actions. It also proves a more general foliated version of local 
rigidity, which is crucially used to prove local rigidity for quasi-affine actions of higher rank semisimple Lie groups and their lattices in a later work.

When $\Gamma$ is a lattice in a simple Lie group of rank at least 2 , a weaker version of this result was proved by Zimmer in 1985 for ergodic, volume preserving perturbations of an ergodic, isometric actions of such $\Gamma$, and the local rigidity was proved by Benveniste [Ben] in 2000 for smooth actions. It is well-known that such higher rank lattices have property $(\mathrm{T})$. Besides strengthening these results, another point of the paper [05-3] is to derive the local rigidity from the property (T) of the discrete groups $\Gamma$ alone, without using structures of the ambient Lie groups.

By definition, a locally compact, $\sigma$-compact group $\Gamma$ has property $(\mathrm{T})$ if any continuous isometric action of $\Gamma$ on a Hilbert space has a fixed point. The paper [05-3] gives a generalization of this standard fixed point property to a wider class of actions, i.e., this fixed point property persists for actions which are perturbations of isometric actions.

Using results in [03] and [05-3], a very general local rigidity result about actions of semisimple Lie groups $G$ with all simple factors of real rank at least two and their lattices $\Gamma$ are proved in [06-2]. One special case says that any action of $\Gamma$ by toral automorphisms is locally rigid. More generally, given a manifold $M$ on which $\Gamma$ acts isometrically and a torus $T^{n}$ on which it acts by automorphisms, the diagonal action on $T^{n} \times M$ is locally rigid.

Though the standard actions defined above are natural and attractive, they are in the process of being replaced by affine algebraic actions and generalized affine algebraic actions. They provide the natural languages to describe results in [03] [05-3], which are stronger than forms presented above. For more details about the current status of the local rigidity, see the survey article by Fisher [Fi].

\section{RANDOM WALKS ON HOMOGENEOUS SPACES AND REDUCTION THEORY}

An important question for a random walk is to decide if it is transient or recurrent. It is well-known that the random walks on $\mathbb{Z}^{2}$ are recurrent but they are transient on $\mathbb{Z}^{n}$ for $n \geq 3$. There are also quantitative versions of these properties.

Motivated by the results in [Da1-2] [75-2] on recurrence properties of orbits of one parameter unipotent subgroups, the joint paper with Eskin [04-2] studies recurrence properties of random walks on finite volume homogeneous manifolds. Specifically, let $G$ be a linear semisimple Lie group, $\Gamma$ a nonuniform irreducible lattice in $G$, and $\mu$ a probability measure on $G$. Then $\mu$ defines a random walk on the noncompact space $G / \Gamma$, which is recurrent. 
Let $\mu^{(n)}$ be the convolution of $\mu$ with itself $n$ times, and $\delta_{x}$ be the probability measure supported on $x \in G / \Gamma$. Then one of the main results of the paper [04-2] is as follows. Suppose that the noncompact part of the Zariski closure of the subgroup generated by $\operatorname{supp}(\mu)$ is semisimple and is not contained in any conjugate of a proper $\Gamma$-rational parabolic subgroup of $G$. Then for every compact set $C \subset G / \Gamma$ and every $\varepsilon>0$, there exists a compact set $K$ such that for every $x \in C$ and all $n>0$, the following inequality holds:

$$
\mu^{(n)} * \delta_{x}(K)>1-\varepsilon .
$$

Similarly, for every $\varepsilon>0$, there exists a compact set $K$ such that for every $x \in G / \Gamma$ and all sufficiently large $n$, the above inequality also holds.

One application of these results is a new proof of a special case of a fundamental result for arithmetic subgroups $\Gamma$ of semisimple linear algebraic groups $\mathbf{G}$, i.e., the volume of the quotient $\Gamma \backslash G$ is finite with respect to every invariant measure on $G=\mathbf{G}(\mathbb{R})$. This was proved by Borel and Harish-Chandra $[\mathrm{BoHC}]$ as a corollary of the reduction theory for arithmetic subgroups developed by them.

Specifically, this finite volume result was proved in [04-2] under the assumption there exists a faithful representation of $\mathbf{G}$ which is defined over $\mathbb{Q}$ and is irreducible over $\mathbb{R}$.

Another new proof of this finiteness of the volume of $\Gamma \backslash G$ for general arithmetic subgroups $\Gamma$ of semisimple groups $\mathbf{G}$ is given in the paper [04-4] using a simplified version of the results [04-2] based on recurrence properties of random walks on the space of lattices in $\mathbb{R}^{n}$.

\section{CoARSE MEtrics ON REDUCTIVE GROUPS}

Let $\mathbf{G}$ be an algebraic group defined over $\mathbb{R}$ and let $G$ be the identity component of the real locus $\mathbf{G}(\mathbb{R})$. For every bounded symmetric open neighbourhood $L$ of the identity element in $G$, one can associate with it a "word metric" $\rho_{L}$ on $G$, defined by

$$
\rho_{L}(g, h)=\min \left\{i \mid g^{-1} h \in L^{i}\right\} .
$$

This metric defines a discrete topology on $G$, but is quasi-isometric to the metric induced from any left-invariant Riemannian metric on $G$. A pseudometric on $G$ is said to be normlike if it is $K$-biinvariant for a maximal compact subgroup $K$ and corresponds to a norm when restricted to a maximal connected diagonalisable subgroup of $G$. The joint paper with Abels [04-3] compares pseudo-metrics on $G$ under a notion of coarse equivalence, motivated by a conjecture of Siegel on comparison of restrictions of invariant metrics to Siegel sets. Specifically, two pseudometrics $\rho_{1}$ and $\rho_{2}$ are said to be coarsely equal if there exists $C>0$ such that

$$
\left|\rho_{1}(g, h)-\rho_{2}(g, h)\right| \leq C \quad \text { for all } g, h \in G .
$$


Then a special case of the main result of [04-3] says that when $G$ is reductive, for any bounded symmetric open neighbourhood $L$ of the identity element in $G$, the associated word metric $\rho$ is coarsely equal to a normlike pseudometric on $G$.

\section{EFFECTIVE ERGODIC THEORY}

As discussed earlier, Margulis has ingeniously used ergodic theory to prove many seemingly unrelated results. A basic aspect of ergodic theory concerns convergence of measures. For example, the basic ergodic theorem says that in an ergodic system, the time average is equal to the space average almost everywhere.

Effective ergodic theory aims to obtain good bounds on the rate of such convergence. Questions of this type on effective ergodic theory, or rather effective proofs of results which are proved using ergodic theory, are natural and have been raised by Margulis in [00-3, pp. 167-169].

For example, in the solutions to the Oppenheim conjecture on the values of quadratic forms $B$ at integral vectors, there are no estimates on the norm of the shortest integral vectors $v \in \mathbb{Z}^{n}$ with values $B(v)$ lying in an interval $(a, b)$.

Another ineffective aspect concerns bounds on the error terms for $N_{(a, b)}^{B}$, the counting function for the integral vectors with values in $(a, b)$ (see $\S 25$ for definition). As mentioned before, the joint paper with Dani [93] together with the joint papers with Eskin and Mozes [95-3] [98-1] [05-1] give sharp bounds and asymptotics for $N_{(a, b)}^{B}$, but no estimates on the error terms are known. One reason for these ineffective results is that the proofs depend on the Ratner results on uniform distribution theorem, which is quite ineffective.

Similar ineffective problems occur in the study of the asymptotic behaviors of the number of lattice points on affine homogeneous varieties [00-3, Problem 6] and in the proofs of the orbit closure theorem and uniform distribution theorem (see [00-3, Problem 7] for precise formulation on desired effective proofs of these results.)

Ratner's classification of invariant ergodic measures of unipotent subgroups $U$ of $G$ on homogeneous spaces $\Gamma \backslash G$ is also crucial to the solution of the Oppenheim conjecture in $\S 24$ but is ineffective. Closely related to this is a result of Mozes and Shah $[\mathrm{MozS}]$ which states that non-zero weak*-limits of $U$-invariant ergodic probability measures are again ergodic. Specifically, let $H \subset G$ be a semisimple subgroup generated by unipotent elements. Then a special case of the results of [MozS] states that the only nonzero weak*-limits of normalized Haar measures on closed $H$-orbits in $\Gamma \backslash G$ are Haar measures on closed orbits of a closed subgroup $S \supseteq H$. 
After these problems were raised in [00-3], some important effective results have been obtained in the past few years. One of the results of the joint paper with Einsiedler and Venkatesh [07-2] gives a polynomial rate convergence of such a sequence of $H$-invariant measures to a Haar measure of an $S$-orbit, under the assumption that $G$ is semisimple and $\Gamma$ is an arithmetic subgroup of $G$.

Closely related to convergence of measures are problems about equi-distribution, for example, distribution of translates of orbits of subgroups. Effective distribution results for Linnik problems are also obtained in [07-2].

See the joint paper of Margulis with Kleinbock [07-1] and the paper [BeO] for related effective results on equistribution of translates of special subgroups and S-integraal points.

\section{Problem lists by Margulis}

Besides problems raised by Margulis in various places, there are two such lists in [75-4, pp. 38-39] and [00-3]. Another list of problems is [Gor], where many problems were suggested by Margulis.

In [75-4], there are three kinds of problems:

(1) Generalizations of results for linear groups to isometry groups of simply connected complete Riemannian manifolds with non-positive sectional curvature.

(2) Existence of non-uniform (non-arithmetic) lattices in semisimple linear real Lie groups of $\mathbb{R}$-rank 1 .

(3) Group structures of higher rank irreducible lattices, in particular arithmetic subgroups of algebraic groups.

Unlike the paper [75-4] which also gives an impressive summary of results on arithmeticity and rigidity of lattices of Lie groups, the paper [00-3] are mainly concerned with problems and conjectures related to rigidity of actions of Lie groups and lattices, and an application to the Littlewood conjecture on sizes of non-integral parts of integral multiples of any two given numbers (see the next page).

More specifically, the paper [00-3] contains the following types of problems:

(1) Orbit closures, invariant measures and uniform distribution on homogeneous spaces.

(2) Application to the Littlewood conjecture.

(3) Effective proofs of the Oppenheim conjecture on values of indefinite irrational quadratic forms at integral points and more generally effective ergodic theory. 
(4) Arithmeticity and super-rigidity for lattices acting on the complex hyperbolic spaces.

(5) Existence of compact quotients of homogeneous spaces.

(6) Classification of actions of higher rank groups and their lattices.

(7) Classification of Anosov diffeomorphisms.

Due to the lack of knowledge of the author, we will only briefly comment on few of the problems raised by Margulis in [75-4] [00-3] (for effective ergodic theeory, see $\S 39)$.

If $\Gamma$ is a finitely generated subgroup of $G L(n, \mathbb{C})$, it is known by Selberg [Se3, Lemma 8, p. 154] that $\Gamma$ admits a torsion-free normal subgroup $\Gamma^{\prime}$ of finite index. This fact is important for many applications and is known as the Selberg Lemma. In [Rag1, Corollary 6.13], a slightly more general result is proved for subgroups of not necessarily linear Lie groups. Specifically, it says that if a connected Lie group $\mathbf{G}$ admits a linear representation $\rho: G \rightarrow G L(n, \mathbb{C})$ such that the kernel of $\rho$ is torsion-free, then any finitely generated subgroup of $G$ admits a torsion-free subgroup $\Gamma^{\prime}$ of finite index. An immediate corollary is that if $X$ is a symmetric space without compact factors and $\operatorname{Isom}(X)$ its isometry group, then any finitely generated subgroup of $I \operatorname{som}(X)$ admits a torsion-free subgroup of finite index. The first question in [75-4, p. 38] asks whether the same conclusion holds if $X$ is replaced by a general complete Riemannian manifold with non-positive sectional curvature.

One reason is that in [Se3] and [Rag1], the fact that the elements of $\Gamma$ can be realized as matrices is crucial for algebraic methods. One point of this question of Margulis is to understand the extent to which the above result on the existence of torsion-free subgroups of finite index only depends on the geometry of $X$ (or on the linear structure of the groups).

If $\Gamma \subset \operatorname{Isom}(X)$ is a discrete subgroup, then $\Gamma$ acts properly on $X$ and the quotient $\Gamma \backslash X$ is an orbifold. In this special case, this question of Margulis is equivalent to if the orbifold $\Gamma \backslash X$ admits a finite smooth cover. In general, not every orbifold admits a finite smooth cover, and one question is how the assumption on the non-positive sectional curvature of $X$ can be used for this purpose. (See $[$ ALR] for references on orbifolds.)

The second problem on [75-4, p.38] is concerned witht existence of (nonarithmetic) nonuniform lattices, motivated by construction of some non-arithmetic lattices acting on the real hyperbolic spaces of low dimension by Makarov and Vinberg, and the arithmeticity theorem of Margulis for irreducible higher rank lattices. The existence of non-arithmetic lattices for all real hyperbolic spaces is established by Gromov and Piatetski-Shapiro in [GPS]. For the complex hyperbolic spaces in complex dimension 2 and 3, non-arithmetic lattices have been constructed by Mostow (see $[\mathrm{DeM}]$ and references there). As mentioned at the 
end of $\S 9$, all lattices for other two rank one symmetric spaces are arithmetic by [Co] and [GS]. Therefore, the remaining cases are for lattices acting on the complex hyperbolic spaces. For lattices acting on the complex hyperbolic spaces, a weaker notion of arithmeticity, called integral, has been studied for them. See $[\mathrm{Ye}]$ and $[\mathrm{Kli}]$ and the survey article $[\mathrm{Sp}]$.

The third question in [75-4, p. 38] asks if any nontrivial normal subgroup of an higher rank irreducible lattice has finite index. This is solved by himself. See $\S 10$.

The sixth question in [75-4, p. 38] asks about proportionality of covolumes of lattices in general semisimple Lie groups. See $\S 23$ for some results on this problem.

The seventh problem in [75-4, p. 39] is concerned with quotients of simply connected complete Riemannian manifolds of nonpositive without any flat factors. One question asks if there is a uniform lower bound on volumes of such quotients if the sectional curvature is uniformly bounded from below. Other problems are concerned with finiteness properties of these quotients if their volumes are bounded from above by a given fixed constant. The Margulis Lemma discussed in $\S 6$ is related to these problems.

The deep work of Ratner proved a conjecture of Raghunathan and generalizations on the orbit closure of unipotent flows on homogeneous manifolds which are quotients of Lie groups by lattices [Ra2]. It is natural to consider flows defined by more general subgroups. The first several conjectures in [00-3] are concerned with conditions under which orbit closures are homogeneous or manifolds, and conditions under which invariant and ergodic measures are algebraic.

For any $x \in \mathbb{R}$, let $\|x\|$ denote the distance $d(x, \mathbb{Z})$ with respect to the usual absolute value on $\mathbb{R}$. Then the Littlewood conjecture states that for any two numbers $\alpha, \beta \in \mathbb{R}$,

$$
\liminf _{n \rightarrow+\infty} n\|n \alpha\|\|n \beta\|=0
$$

It was proved by Cassels and Swinnterton-Dyer in 1955 that the Littlewood conjecture follows from a conjecture that the infimum of the absolute values of $L$ on the integral points $\mathbb{Z}^{n}$ is equal to 0 , where $L$ is the product of $n$ linearly independently linear forms on $\mathbb{R}^{n}$ and is not a multiple of a form with integer coefficients.

It was also observed by Cassels and Swinnterton-Dyer (implicitly) in 1955 for $n=3$ and Margulis [97-3] for general $n$ that this latter conjecture is implied by the following conjecture on the orbit closure: Assume that $n \geq 3$. Let $D \subset S L(n, \mathbb{R})$ be the subgroup of all diagonal matrices. If for any $z \in S L(n, \mathbb{R}) / S L(n, \mathbb{Z})$, the orbit $D z$ in $S L(n, \mathbb{R}) / S L(n, \mathbb{Z})$ is relatively compact, then $D z$ is closed. 
This conjecture is a special case of the first conjecture in [00-3]. This reformulation of, or rather the approach to, the Littlewood conjecture has been quite influential. In [EKL], a partial result towards the Littlewood conjecture that the exception set is of Hausdorff dimension 0 is obtained by classifying measures on $S L(n, \mathbb{R}) / S L(n, \mathbb{Z})$ with positive entropy which are invariant and ergodic under the action of the subgroup of all positive diagonal matrices.

Another type of problems is concerned with orbit closures of semigroups of endomorphisms of nilmanifolds, i.e., to find conditions under which the closure is the finite union of closed homogeneous subspaces, and structures of invariant and ergodic measures. See $[\mathrm{Sp}]$ for a survey and $[\mathrm{KaS}]$ for related results.

Rigidity and classification of actions of higher rank semissimple Lie groups and their lattices are related to the Zimmer program. Several questions of this type are raised in [00-3]. See [Sp] and [GoS] for references and related results.

\section{Partial list of papers and books of Margulis.}

\section{REFERENCES}

[66] G.Margulis, Positive harmonic functions on nilpotent groups, Soviet Math. Dokl. 7 (1966) 241-244.

[67] G.Margulis, Y-flows on three dimensinal manifolds, Russian Math. Surveys 22 (1967) 164-166, an appendix to paper [AS] below.

[68] D.Kazhdan, G.Margulis, A proof of Selberg's hypothesis, Mat. Sb. (N.S.) 75(117) (1968) 163-168.

[69-1] G.Margulis, Discrete subgroups of real semi-simple Lie groups, Mat. Sb. (N.S.) 80(122) (1969) 600-615.

[69-2] G.Margulis, Certain applications of ergodic theory to the investigation of manifolds of negative curvature, Functional Anal. Appl. 3 (1969) 335-336.

[69-3] G.Margulis, On the arithmeticity of discrete groups, Soviet Math. Dokl. 10 (1969) 900902.

[70-1] G.Margulis, The isometry of closed manifolds of constant negative curvature with the same fundamental group, Soviet Math. Dokl. 11 (1970) 722-723.

[70-2] G.Margulis, Certain measures that are connected with Y-flows on compact manifolds, Functional Anal. Appl. 4 (1970) 55-67.

[71] G.Margulis, The action of unipotent groups in a lattice space, Mat. Sb. (N.S.) 86(128) (1971) 552-556.

[73-1] G.Margulis, Arithmeticity of nonuniform lattices, Functional Anal. Appl. 7 (1973) 245246.

[73-2] G.Margulis, Explicit constructions of expanders, Problems of Information Transmission 9 (1973), no. 4, 325-332.

[74-1] G.Margulis, Arithmeticity and finite-dimensional representations of uniform lattices, Functional Anal. Appl. 8 (1974), no. 1, 258-259.

[74-2] G.Margulis, Arithmetic properties of discrete subgroups, Russian Math. Surveys 29 (1974), no. 1, 107-156.

[74-3] G.Margulis, Probabilistic characteristics of graphs with large connectivity, Problems of Information Transmission 10 (1974), no. 2, 174-179. 
[75-1] G.Margulis, Arithmeticity of nonuniform lattices in weakly noncompact groups, Functional Anal. Appl. 9 (1975), no. 1, 31-38.

[75-2] G.Margulis, Non-uniform lattices in semisimple algebraic groups, in Lie groups and their representations (Proc. Summer School on Group Representations of the Bolyai János Math. Soc., Budapest, 1971), pp. 371-553. Halsted, New York, 1975.

[75-3] G.Margulis, On the action of unipotent groups in the space of lattices, in Lie groups and their representations (Proc. Summer School, Bolyai, János Math. Soc., Budapest, 1971), pp. 365-370. Halsted, New York, 1975.

[75-4] G.Margulis, Discrete groups of motions of manifolds of nonpositive curvature, Proceedings of the International Congress of Mathematicians (Vancouver, B.C., 1974), Vol. 2, pp. 21-34. Canad. Math. Congress, Montreal, Que., 1975. Amer. Math. Soc. Tranasl. 109, pp. 33-45.

[77-1] G.Margulis, Cobounded subgroups in algebraic groups over local fields, Functional Anal. Appl. 11 (1977) 119-122.

[77-2] G.Margulis, Arithmeticity of irreducible lattices in semisimple groups of rank greater than 1, an appendix to the Russian translation of the book Discrete subgroups of Lie groups by M.S.Raghunathan, 1977.

[77-3] G.Margulis, G.Soifer, A criterion for the existence of maximal subgroups of infinite index in a finitely generated linear group, Soviet Math. Dokl. 18 (1977), no. 3, 847-851.

[78-1] G.Margulis, Factor-groups of discrete subgroups, Soviet Math. Dokl. 19 (1978), no. 5, 1145-1149.

[78-2] G.Margulis, Factor groups of discrete subgroups and measure theory, Functional Anal. Appl. 12 (1978), no. 4, 295-305.

[79-1] G.Margulis, Finiteness of quotient groups of discrete subgroups, Functional Anal. Appl. 13 (1979) 178-187.

[79-2] G.Margulis, G.Soifer, Nonfree maximal subgroups of infinite index of the group $\mathrm{SL}_{n}(Z)$, Russian Math. Surveys 34 (1979), no. 4, 178-179.

[80-1] G.Margulis, Multiplicative groups of a quaternion algebra over a global field, Soviet Math. Dokl. 21 (1980), no. 3, 780-784.

[80-2] G.Margulis, Some remarks on invariant means, Monatsh. Math. 90 (1980) 233-235.

[81-1] G.Margulis, G.Soifer, Maximal subgroups of infinite index in finitely generated linear groups, J. Algebra 69 (1981) 1-23.

[81-2] G.Margulis, On the decomposition of discrete subgroups into amalgams. Selected translations, Selecta Math. Soviet. 1 (1981) 197-213.

[82-1] G.Margulis, Explicit constructions of graphs without short cycles and low density codes, Combinatorica 2 (1982) 71-78.

[82-2] G.Margulis, Finitely-additive invariant measures on Euclidean spaces, Ergodic Theory Dynam. Systems 2 (1982) 383-396.

[83-1] G.Margulis, Free completely discontinuous groups of affine transformations, Soviet Math. Dokl. 28 (1983) 435-439.

[83-2] A.Huckleberry, G.Margulis, Invariant analytic hypersurfaces, Invent. Math. 71 (1983) 235-240.

[84-1] G.Margulis, Arithmeticity of the irreducible lattices in the semisimple groups of rank greater than 1, Invent. Math. 76 (1984) 93-120.

[84-2] G.Margulis, Complete affine locally flat manifolds with a free fundamental group, Zap. Nauchn. Sem. Leningrad. Otdel. Mat. Inst. Steklov. (LOMI) 134 (1984) 190-205.

[84-3] G.Margulis, A.Omelchenko, Deformation of plane curves, Problemy Peredachi Informatsii 20 (1984) 31-40.

[84-4] G.Margulis, Arithmetic groups and graphs without short cycles, in 6th International Symp. on information theory, Tashkent 1984, Abstracts, Vol. 1, pp. 123-125. 
[86] G.Margulis, J.Rohlfs, On the proportionality of covolumes of discrete subgroups, Math. Ann. 275 (1986) 197-205.

[87-1] G.Margulis, Formes quadratriques indéfinies et flots unipotents sur les espaces homogènes, C. R. Acad. Sci. Paris Sér. I Math. 304 (1987) 249-253.

[87-2] I.Goldsheid, G.Margulis, The condition of simplicity for the spectrum of Lyapunov exponents, Soviet Math. Dokl. 35 (1987) 309-313.

[88-1] G.Margulis, Explicit group-theoretic constructions of combinatorial schemes and their applications in the construction of expanders and concentrators, Problems Inform. Transmission 24 (1988) 39-46.

[88-2] G.Margulis, Lie groups and ergodic theory, in Algebra-some current trends, pp. 130146, Lecture Notes in Math., 1352, Springer, 1988.

[89-1] F.Grunewald, G.Margulis, Transitive and quasitransitive actions of affine groups preserving a generalized Lorentz-structure, J. Geom. Phys. 5 (1988) 493-531 (1989).

[89-2] S.Dani, G.Margulis, Values of quadratic forms at primitive integral points, C. R. Acad. Sci. Paris Sér. I Math. 308 (1989) 199-203.

[89-3] G.Margulis, Discrete subgroups and ergodic theory, in Number theory, trace formulas and discrete groups, pp. 377-398, Academic Press, 1989.

[89-4] S.Dani, G.Margulis, Values of quadratic forms at primitive integral points, Invent. Math. 98 (1989) 405-424.

[89-5] I.Goldsheid, G.Margulis, Lyapunov exponents of a product of random matrices, Russian Math. Surveys 44 (1989), no. 5, 11-71.

[89-6] G.Margulis, Indefinite quadratic forms and unipotent flows on homogeneous spaces, in Dynamical systems and ergodic theory, pp. 399-409, Banach Center Publ., 23, PWN, Warsaw, 1989.

[90-1] S.Dani, G.Margulis, Orbit closures of generic unipotent flows on homogeneous spaces of SL(3, R), Math. Ann. 286 (1990) 101-128.

[90-2] S.Dani, G.Margulis, Values of quadratic forms at integral points: an elementary approach, Enseign. Math. (2) 36 (1990) 143-174.

[90-3] G.Margulis, Orbits of group actions and values of quadratic forms at integral points, in Festschrift in honor of I. I. Piatetski-Shapiro on the occasion of his sixtieth birthday, Part II, pp. 127-150, Israel Math. Conf. Proc., 3, Weizmann, Jerusalem, 1990.

[91-1] G.Margulis, Discrete subgroups of semisimple Lie groups, Ergebnisse der Mathematik und ihrer Grenzgebiete (3), 17. Springer-Verlag, Berlin, 1991. x+388 pp.

[91-2] G.Margulis, Compactness of minimal closed invariant sets of actions of unipotent groups, Geom. Dedicata 37 (1991) 1-7.

[91-3] S.Dani, G.Margulis, Asymptotic behaviour of trajectories of unipotent flows on homogeneous spaces, Proc. Indian Acad. Sci. Math. Sci. 101 (1991) 1-17.

[91-4] G.Margulis, Dynamical and ergodic properties of subgroup actions on homogeneous spaces with applications to number theory, Proceedings of the International Congress of Mathematicians, Vol. I, II (Kyoto, 1990), pp. 193-215, Math. Soc. Japan, Tokyo, 1991.

[92-1] S.Dani, G.Margulis, On the limit distributions of orbits of unipotent flows and integral solutions of quadratic inequalities, C. R. Acad. Sci. Paris Sér. I Math. 314 (1992) 699704 .

[92-2] G.Margulis, G.Tomanov, Measure rigidity for algebraic groups over local fields, C. R. Acad. Sci. Paris Sér. I Math. 315 (1992) 1221-1226.

[93] S.Dani, G.Margulis, Limit distributions of orbits of unipotent flows and values of quadratic forms, in I. M. Gelfand Seminar, pp. 91-137, Adv. Soviet Math., 16, Part 1, Amer. Math. Soc., 1993. 
[94] G.Margulis, G.Tomanov, Invariant measures for actions of unipotent groups over local fields on homogeneous spaces, Invent. Math. 116 (1994) 347-392.

[95-1] G.Margulis, G.Mostow, The differential of a quasi-conformal mapping of a CarnotCarathéodory space, Geom. Funct. Anal. 5 (1995) 402-433.

[95-2] H.Abels, G.Margulis, G.Soifer, Semigroups containing proximal linear maps, Israel J. Math. 91 (1995) 1-30.

[95-3] A.Eskin, G.Margulis, S.Mozes, On a quantitative version of the Oppenheim conjecture, Electron. Res. Announc. Amer. Math. Soc. 1 (1995) 124-130.

[96-1] D.Kleinbock, G.Margulis, Bounded orbits of nonquasiunipotent flows on homogeneous spaces, in Sină̈'s Moscow Seminar on Dynamical Systems, pp. 141-172, Amer. Math. Soc. Transl. Ser. 2, 171, Amer. Math. Soc., 1996.

[96-2] G.Margulis, G.Tomanov, Measure rigidity for almost linear groups and its applications, J. Anal. Math. 69 (1996) 25-54.

[97-1] H.Abels, G.Margulis, G.Soifer, Properly discontinuous groups of affine transformations with orthogonal linear part, C. R. Acad. Sci. Paris Sér. I Math. 324 (1997) 253-258.

[97-2] G.Margulis, Existence of compact quotients of homogeneous spaces, measurably proper actions, and decay of matrix coefficients, Bull. Soc. Math. France 125 (1997) 447-456.

[97-3] G.Margulis, Oppenheim conjecture, in Fields Medallists' Lectures, pp. 272-327, World Sci. Ser. 20th Century Math., vol. 5, World Sci. Publ., 1997.

[98-1] A.Eskin, G.Margulis, S.Mozes, Upper bounds and asymptotics in a quantitative version of the Oppenheim conjecture, Ann. of Math. (2) 147 (1998) 93-141.

[98-2] D.Kleinbock, G.Margulis, Flows on homogeneous spaces and Diophantine approximation on manifolds, Ann. of Math. (2) 148 (1998) 339-360.

[98-3] G.Margulis, S.Mozes, Aperiodic tilings of the hyperbolic plane by convex polygons, Israel J. Math. 107 (1998) 319-325.

[99-1] D.Kleinbock, G.Margulis, Logarithm laws for flows on homogeneous spaces, Invent. Math. 138 (1999) 451-494.

[99-2] A.Karlsson, G.Margulis, A multiplicative ergodic theorem and nonpositively curved spaces, Comm. Math. Phys. 208 (1999) 107-123.

[00-1] G.Margulis, E.Vinberg, Some linear groups virtually having a free quotient, J. Lie Theory 10 (2000) 171-180.

[00-2] G.Margulis, A.Nevo, E.Stein, Analogs of Wiener's ergodic theorems for semisimple Lie groups. II, Duke Math. J. 103 (2000) 233-259.

[00-3] G.Margulis, Problems and conjectures in rigidity theory, in Mathematics: frontiers and perspectives, pp. 161-174, Amer. Math. Soc., 2000.

[00-4] G.Margulis, G.Mostow, Some remarks on the definition of tangent cones in a CarnotCarathéodory space, J. Anal. Math. 80 (2000) 299-317.

[00-5] W.Goldman, G.Margulis, Flat Lorentz 3-manifolds and cocompact Fuchsian groups, in Crystallographic groups and their generalizations, pp. 135-145, Contemp. Math., 262, Amer. Math. Soc., 2000.

[00-6] G.Margulis, Free subgroups of the homeomorphism group of the circle, C. R. Acad. Sci. Paris Sér. I Math. 331 (2000) 669-674.

[01-1] G.Margulis, N.Qian, Rigidity of weakly hyperbolic actions of higher real rank semisimple Lie groups and their lattices, Ergodic Theory Dynam. Systems 21 (2001) 121-164.

[01-2] V.Bernik, D.Kleinbock, G.Margulis, Khintchine-type theorems on manifolds: the convergence case for standard and multiplicative versions, Internat. Math. Res. Notices 9 (2001) 453-486.

[02-1] H.Abels, G.Margulis, G.Soifer, On the Zariski closure of the linear part of a properly discontinuous group of affine transformations, J. Differential Geom. 60 (2002) 315-344. 
[02-2] V.Beresnevich, V.Bernik, D.Kleinbock, G.Margulis, Metric Diophantine approximation: the Khintchine-Groshev theorem for nondegenerate manifolds, Mosc. Math. J. 2 (2002) 203-225.

[02-3] G.Margulis, Diophantine approximation, lattices and flows on homogeneous spaces, in $A$ panorama of number theory or the view from Baker's garden, pp. 280-310, Cambridge Univ. Press, 2002.

[03] D.Fisher, G.Margulis, Local rigidity for cocycles, in Surveys in differential geometry, Vol. VIII, pp.191-234, Int. Press, 2003.

[04-1] G.Margulis, On some aspects of the theory of Anosov systems, With a survey by Richard Sharp: Periodic orbits of hyperbolic flows, Springer Monographs in Mathematics. Springer-Verlag, 2004. vi+139 pp.

[04-2] A.Eskin, G.Margulis, Recurrence properties of random walks on finite volume homogeneous manifolds, Random walks and geometry, pp. 431-444, Walter de Gruyter GmbH \& Co. KG, Berlin, 2004.

[04-3] H.Abels, G.Margulis, Coarsely geodesic metrics on reductive groups, in Modern dynamical systems and applications, pp. 163-183, Cambridge Univ. Press, 2004.

[04-4] G.Margulis, Random walks on the space of lattices and the finiteness of covolumes of arithmetic subgroups, in Algebraic groups and arithmetic, pp. 409-425, Tata Inst. Fund. Res., Mumbai, 2004.

[05-1] A.Eskin, G.Margulis, S.Mozes, Quadratic forms of signature $(2,2)$ and eigenvalue spacings on rectangular 2-tori, Ann. of Math. (2) 161 (2005) 679-725.

[05-2] H.Abels, G.Margulis, G.Soifer, The Auslander conjecture for groups leaving a form of signature $(n-2,2)$ invariant, Israel J. Math. 148 (2005) 11-21.

[05-3] D.Fisher, G.Margulis, Almost isometric actions, property (T), and local rigidity, Invent. Math. 162 (2005) 19-80.

[06-1] W.Goldman, F.Labourie, G.Margulis, Proper affine actions and geodesic flows of hyperbolic surfaces, arXiv:math/0406247.

[06-2] D.Fisher, G.Margulis, Local rigidity of affine actions of higher rank groups and lattices, arXiv:math/0408213.

[06-3] T.Gelander, A.Karlsson, G.A.Margulis, Superrigidity, generalized harmonic maps and uniformly convex spaces, arXiv:math/0606256v2.

[07-1] D.Kleinbock, G.Margulis, On effective equidistribution of expanding translates of certain orbits in the space of lattices, preprint, arXiv:math.DS/0702433.

[07-2] M.Einsiedler, G.Margulis, A.Venkatesh, Effective equidistribution of closed orbits of semisimple groups on homogeneous spaces, preprint, 2007.

\section{Papers and books by others cited in this article.}

\section{REFERENCES}

[Ah] D.Ahiezer, Invariant meromorphic functions on complex semisimple Lie groups, Invent. Math. 65 (1981/82), no. 3, 325-329.

[ALR] A.Alejandro, J.Leida, Y.Ruan, Orbifolds and Stringy Topology, Cambridge Tracts in Mathematics, vol. 171, Cambridge University Press, 2007.

[Al] R.Alperin, Locally compact groups acting on trees and property T, Monatsh. Math. 93 (1982) 261-265.

[AmGS] R.Ammann, B.Grünbaum, G.Shephard, Aperiodic tiles, Discrete Comput. Geom. 8 (1992) 1-25. 
[AS] D.Anosov, J.Sinai, Certain smooth ergodic systems, Russian Math. Surveys 22 (1967) 103-167.

[At] J.Athreya, Quantitative recurrence and large deviations for Teichmuller geodesic flow, Geom. Dedicata 119 (2006) 121-140.

[Bab] M.Babillot, An introduction to Poisson boundaries of Lie groups, in Probability measures on groups: recent directions and trends, pp. 1-90, Tata Inst. Fund. Res., 2006.

[BaS] U.Bader, Y.Shalom, Factor and normal subgroup theorems for lattices in products of groups, Invent. Math. 163 (2006) 415-454.

[BaGS] W.Ballmann, M.Gromov, V.Schroeder, Manifolds of nonpositive curvature, Progress in Mathematics, 61. Birkhäuser, 1985. vi+263 pp.

[Bea] A.Beardon, The geometry of discrete groups, GTM 91, Springer, 1983.

[BeO] Y.Benoist, H.Oh, Effective equidistribution of S-integral points on symmetric varieties, preprint, 2007.

[Ben] E.Benveniste, Rigidity of isometric lattice actions on compact Riemannian manifolds, Geom. Funct. Anal. 10 (2000) 516-542.

[BeB] V.Beresnevich, V.Bernik, On a metrical theorem of W. Schmidt, Acta Arith. 75 (1996) 219-233.

[BFH1] M.Bestvina, M.Feighn, M.Handel, The Tits alternative for $\operatorname{Out}\left(F_{n}\right)$. I. Dynamics of exponentially-growing automorphisms, Ann. of Math. 151 (2000) 517-623.

[BFH2] M.Bestvina, M.Feighn, M.Handel, The Tits alternative for $\operatorname{Out}\left(F_{n}\right)$. II. A Kolchin type theorem, Ann. of Math. 161 (2005) 1-59.

[BlW] J.Block, S.Weinberger, Aperiodic tilings, positive scalar curvature and amenability of spaces, J. Amer. Math. Soc. 5 (1992) 907-918.

[Bo1] A.Borel, Commensurability classes and volumes of hyperbolic 3-manifolds, Ann. Scuola Norm. Sup. Pisa Cl. Sci. 8 (1981) 1-33.

[Bo2] A.Borel, Sous-groups discrets de groupes semi-simples (d'aprè D.Kajdan et G.A.Margoulis, Séminaire Bourebaki, Exp. 358 (1968/69). Lect. Notes Math. 179 (1971) 199-216.

[Bo3] A.Borel, On the work of M. S. Raghunathan, in Algebraic groups and arithmetic, pp.124, Tata Inst. Fund. Res., 2004.

[BoHC] A.Borel, Harish-Chandra, Arithmetic subgroups of algebraic groups, Ann. of Math. 75 (1962) 485-535.

[BoP] A.Borel, G.Prasad, Values of isotropic quadratic forms at S-integral points, Compositio Math. 83 (1992) 347-372.

[Bur] M.Burger, Rigidity properties of group actions on CAT(0)-spaces, Proceedings of the International Congress of Mathematicians, Vol. 1, 2 (Zrich, 1994), pp. 761-769, Birkhäuser, 1995.

[BuM1] M.Burger, S.Mozes, Lattices in product of trees, Inst. Hautes Études Sci. Publ. Math. No. 92 (2000) 151-194.

[BuM2] M.Burger, S.Mozes, Groups acting on trees: from local to global structure, Inst. Hautes Études Sci. Publ. Math. No. 92 (2000) 113-150.

[Ca] E.Calabi, On compact, Riemannian manifolds with constant curvature. I, Proc. Sympos. Pure Math., Vol. III, pp. 155-180, Amer. Math. Soc., 1961.

$[\mathrm{CaV}]$ E.Calabi, E.Vesentini, On compact, locally symmetric Khler manifolds, Ann. of Math. 71 (1960) 472-507.

[CaR] P.Caprace, B.Rémy, Simplicité abstraite des groupes de Kac-Moody non affines, C. R. Math. Acad. Sci. Paris 342 (2006) 539-544.

[Cl] L.Clozel, Automorphic forms and the distribution of points on odd-dimensional spheres, Israel J. Math. 132 (2002) 175-187. 
[Co] K.Corlette, Archimedean superrigidity and hyperbolic geometry, Ann. of Math. 135 (1992) 165-182.

[DaH] E.Damek, A.Hulanicki, Asymptotic behavior of the invariant measure for a diffusion related to an NA group, Colloq. Math. 104 (2006) 285-309.

[Da1] S.Dani, On orbits of unipotent flows on homogeneous spaces, Ergodic Theory Dynam. Systems 4 (1984) 25-34.

[Da2] S.Dani, On invariant measures, minimal sets and a lemma of Margulis, Invent. Math. 51 (1979) 239-260.

[Da3] S.Dani, Bounded orbits of flows on homogeneous spaces, Comment. Math. Helv. 61 (1986) 636-660.

[Da4] S.Dani, Flows on homogeneous spaces and Diophantine approximation, Proceedings of the International Congress of Mathematicians, Vol. 1, 2, pp. 780-789, Birkhäuser, 1995.

[Da5] S.Dani, Divergent trajectories of flows on homogeneous spaces and Diophantine approximation, J. Reine Angew. Math. 359 (1985) 55-89.

[Da6] S.Dani, Invariant measures and minimal sets of horospherical flows, Invent. Math. 64 (1981) 357-385.

[Da7] S.Dani, Flows on homogeneous spaces: a review, in Ergodic theory of $Z^{d}$ actions, pp. 63-112, London Math. Soc. Lecture Note Ser., 228, Cambridge Univ. Press, 1996.

[Da8] S.G.Dani, Orbits of horospherical flows, Duke Math. J. 53 (1986) 177-188.

[DeM] P.Deligne, G.Mostow, Commensurabilities among lattices in PU $(1, n)$, Annals of Mathematics Studies, 132, Princeton University Press, 1993. viii+183 pp.

[Dr] V.Drinfeld, Finitely-additive measures on $S^{2}$ and $S^{3}$, invariant with respect to rotations, Functional Anal. Appl. 18 (1984), no. 3, 245-246.

[DuT] N.Dunfield, W.Thurston, Finite covers of random 3-manifolds, Invent. Math. 166 (2006) $457-521$.

[Dy] E.Dynkin, Markov processes and problems in analysis, Proc. Internat. Congr. Mathematicians (Stockholm, 1962) pp. 36-58 Inst. Mittag-Leffler, 1963.

[EKL] M.Einsiedler, A.Katok, E.Lindenstrauss, Invariant measures and the set of exceptions to Littlewood's conjecture, Ann. of Math. 164 (2006) 513-560.

[ElM] N.Elkies, C.McMullen, Gaps in $\sqrt{n} \bmod 1$ and ergodic theory, Duke Math. J. 123 (2004) 95-139

[Es1] A.Eskin, Counting problems and semisimple Lie groups, Prof. of ICM, 1998, vol. 2, pp. 539-552.

[Es2] A.Eskin, Counting problems in moduli space, in Handbook of dynamical systems, Vol. 1B, pp.581-595, Elsevier, 2006.

[EsM] A.Eskin, H.Masur, Asymptotic formulas on flat surfaces, Ergodic Theory Dynam. Systems 21 (2001) 443-478.

[EsMS] A.Eskin, H.Masur, M.Schmoll, Billiards in rectangles with barriers, Duke Math. J. 118 (2003) 427-463.

[EMS] A.Eskin, S.Mozes, N.Shah, Unipotent flows and counting lattice points on homogeneous varieties, Ann. of Math. (2) 143 (1996) 253-299.

[Fa] F.T.Farrell, The Borel conjecture, in Topology of high-dimensional manifolds, pp. 225298, ICTP Lect. Notes, 9, Abdus Salam Int. Cent. Theoret. Phys., Trieste, 2002.

[Fi] D.Fisher, Local Rigidity: Past, Present, Future, to appear Dynamics, ergodic theory and geometry, edited by B.Hasselblatt, an MSRI volume resulting from Katok birthday conference.

[Fu] K.Fukaya, Margulis' lemma in Riemannian geometry, Sugaku Expositions 6 (1993), no. 2, 201-219.

[Fur] A.Furman, Mostow-Margulis rigidity with locally compact targets, Geom. Funct. Anal. 11 (2001) 30-59. 
[GaJS] A.Gamburd, D.Jakobson, P.Sarnak, Spectra of elements in the group ring of SU(2), J. Eur. Math. Soc. (JEMS) 1 (1999) 51-85.

[Gao] Y.Gao, Superrigidity for homomorphisms into isometry groups of CAT(-1) spaces, Transform. Groups 2 (1997) 289-323.

[GhS] É.Ghys,V.Sergiescu, Sur un groupe remarquable de difféomorphismes du cercle, Comment. Math. Helv. 62 (1987) 185-239.

[GoS] E.Goetze, R.Spatzier, Smooth classification of Cartan actions of higher rank semisimple Lie groups and their lattices, Ann. of Math. 150 (1999) 743-773.

[Gor] A.Gorodnik, Open problems in dynamics and related fields, J.Mod. Dyn. 1 (2007) 1-35.

[GO] A.Gorodnik, H.Oh, Orbits of discrete subgroups on a symmetric space and the Furstenberg boundary, To appear in Duke Math. J.

[Gr1] M.Gromov, Almost flat manifolds, J. Differential Geom. 13 (1978) 231-241.

[Gr2] M.Gromov, Groups of polynomial growth and expanding maps, Inst. Hautes Études Sci. Publ. Math. No. 53 (1981) 53-73.

[Gr3] M.Gromov, Asymptotic invariants of infinite groups, in Geometric group theory, Vol. 2 (Sussex, 1991), pp.1-295, London Math. Soc. Lecture Note Ser., vol. 182, Cambridge Univ. Press, 1993.

[GPS] M.Gromov, Piatetski-Shapiro, Nonarithmetic groups in Lobachevsky spaces, Inst. Hautes Études Sci. Publ. Math. No. 66 (1988) 93-103.

[GS] M.Gromov, R.Schoen, Harmonic maps into singular spaces and p-adic superrigidity for lattices in groups of rank one, Inst. Hautes Itudes Sci. Publ. Math. 76 (1992) 165-246.

[Gu] Y.Guivarc'h, Produits de matrices aléatoires et applications aux propriétés géométriques des sous-groupes du groupe linéaire, Ergodic Theory Dynam. Systems 10 (1990) 483-512.

[Hej] D.Hejhal, The Selberg trace formula for $\operatorname{PSL}(2, \mathbb{R})$, Vol. I, Lecture Notes in Mathematics, Vol. 548, Springer, 1976. vi+516 pp.

$[\mathrm{HeH}] \quad$ J.Heinonen, I.Holopainen, Quasiregular maps on Carnot groups, J. Geom. Anal. 7 (1997) $109-148$.

[Ho] R.Howe, A century of Lie theory, in American Mathematical Society centennial publications, Vol. II (Providence, RI, 1988), pp. 101-320, Amer. Math. Soc., 1992.

[Iv] N.Ivanov, Algebraic properties of the Teichmller modular group, Soviet Math. Dokl. 29 (1984) 288-291.

[Ji1] L.Ji, Arithmetic groups and their generalizations: what, why and how, preprint, 2007.

[Ji2] L.Ji, Buildings and their applications in geometry and topology, Asian J. Math. 10 (2006) $11-80$.

[JoY1] J.Jost, S.T.Yau, Harmonic maps and rigidity theorems for spaces of nonpositive curvature, Comm. Anal. Geom. 7 (1999) 681-694.

[JoY2] J.Jost, S.T.Yau, Harmonic maps and superrigidity, in Differential geometry: partial differential equations on manifolds, pp. 245-280, Proc. Sympos. Pure Math., 54, Part 1, Amer. Math. Soc., 1993..

$[\mathrm{KaH}]$ A.Katok, B.Hasselblatt, Introduction to the modern theory of dynamical systems, Encyclopedia of Mathematics and its Applications, 54. Cambridge University Press, 1995, xviii+802 pp.

[KaS] A.Katok, R.Spatzier, Invariant measures for higher-rank hyperbolic abelian actions, Ergodic Theory Dynam. Systems 16 (1996) 751-778.

[Ke] H.Kesten, What is ... percolation? Notices Amer. Math. Soc. 53 (2006), no. 5, 572-573.

[Kl] D.Kleinbock, An extension of quantitative nondivergence and applications to Diophantine exponents, to appear in Trans. AMS.

[KlT] D.Kleinbock, G.Tomanov, Flows on S-arithmetic homogeneous spaces and applications to metric Diophantine approximation, Comm. Math. Helv. 82 (2007) 519-581. 
[Kli] B.Klingler, Sur la rigidité de certains groupes fondamentaux, l'arithméticité des réseaux hyperboliques complexes, et les "faux plans projectifs", Invent. Math. 153 (2003) 105-143.

[KoY] T.Kobayashi, T.Yoshino, Compact Clifford-Klein forms of symmetric spaces-revisited, Pure Appl. Math. Q. 1 (2005) 591-663.

[KoR1] A.Koranyi, H.Reimann, Quasiconformal mappings on the Heisenberg group, Invent. Math. 80 (1985) 309-338.

[KoR2] A.Koranyi, H.Reimann, Foundations for the theory of quasiconformal mappings on the Heisenberg group, Adv. Math. 111 (1995) 1-87.

[Kos] J.Koszul, Rigidité forte des espaces riemanniens localement symétriques (d'après G. D. Mostow), Séminaire Bourbaki (1974/1975), Exp. No. 468, pp. 223-237. Lecture Notes in Math., Vol. 514, Springer, 1976.

[Lan] R.Langlands, On the functional equations satisfied by Eisenstein series, Lecture Notes in Mathematics, Vol. 544, Springer-Verlag, 1976, v+337 pp.

[Li1] L.Lifschitz, Arithmeticity of rank-1 lattices with dense commensurators in positive characteristic, J. Algebra 261 (2003) 44-53.

[Li2] L.Lifschitz, Superrigidity theorems in positive characteristic, J. Algebra 229 (2000) 375404.

[Lu1] A.Lubotzky, Lattices in rank one Lie groups over local fields, Geom. Funct. Anal. 1 (1991) 406-431.

[Lu2] A.Lubotzky, Free quotients and the first Betti number of some hyperbolic manifolds, Transform. Groups 1 (1996) 71-82.

[Lu3] A.Lubotzky, Discrete groups, expanding graphs and invariant measures, Progress in Mathematics, 125. Birkhäuser, 1994. xii+195 pp.

[LuPS] A.Lubotzky, P.Phillips, P.Sarnak, Ramanujan graphs, Combinatorica 8 (1988) 261-277.

[Mc] J.McCarthy, A "Tits-alternative" for subgroups of surface mapping class groups, Trans. Amer. Math. Soc. 291 (1985) 583-612.

[Mi] J.Milnor, A note on curvature and fundamental group, J. Differential Geometry 2 (1968) $1-7$.

[MSY] N.Mok, Y.Siu, S.Yeung, Geometric superrigidity, Invent. Math. 113 (1993) 57-83.

[Mon] N.Monod, Superrigidity for irreducible lattices and geometric splitting, J. Amer. Math. Soc. 19 (2006) 781-814.

[Mor] D.Morris, Ratner's theorems on unipotent flows, Chicago Lectures in Mathematics. University of Chicago Press, 2005. xii+203 pp.

[Mos1] G.Mostow, Strong rigidity of locally symmetric spaces, Annals of Mathematics Studies, No. 78. Princeton University Press, 1973. v+195 pp.

[Mos2] G.Mostow, Quasi-conformal mappings in $n$-space and the rigidity of hyperbolic space forms, Inst. Hautes Études Sci. Publ. Math. No. 34 (1968) 53-104.

[Mos3] G.Mostow, The rigidity of locally symmetric spaces, Actes du Congrès International des Mathématiciens (Nice, 1970), Tome 2, pp. 187-197. Gauthier-Villars, Paris, 1971.

[MoT] G.Mostow, T.Tamagawa, On the compactness of arithmetically defined homogeneous spaces, Ann. of Math. 76 (1962) 446-463.

[Moz1] S.Mozes, Products of trees, lattices and simple groups, Proceedings of the International Congress of Mathematicians, Vol. II (Berlin, 1998). Doc. Math. 1998, Extra Vol. II, pp. 571-582.

[Moz2] S.Mozes, Aperiodic tilings, Invent. Math. 128 (1997) 603-611.

[MozS] S.Mozes, N.Shah, On the space of ergodic invariant measures of unipotent flows, Ergodic Theory Dynam. Systems 15 (1995) 149-159.

[NeZ1] A.Nevo, R.Zimmer, A structure theorem for actions of semisimple Lie groups, Ann. of Math. 156 (2002) 565-594. 
[NeZ2] A.Nevo, R.Zimmer, Homogenous projective factors for actions of semi-simple Lie groups, Invent. Math. 138 (1999) 229-252.

[Oh1] H.Oh, Tempered subgroups and representations with minimal decay of matrix coefficients, Bull. Soc. Math. France 126 (1998) 355-380.

[Oh2] H.Oh, Uniform pointwise bounds for matrix coefficients of unitary representations and applications to Kazhdan constants, Duke Math. J. 113 (2002) 133-192.

[Oh3] H.Oh, Adelic version of Margulis arithmeticity theorem, Math. Ann. 321 (2001) 789-815.

[Oh4] H.Oh, Discrete subgroups generated by lattices in opposite horospherical subgroups, J. Algebra 203 (1998) 621-676.

[Oh5] H.Oh, The Ruziewicz problem and distributing points on homogeneous spaces of a compact Lie group, Israel J. Math. 149 (2005) 301-316.

[OhW] H.Oh, D.Witte, New examples of compact Clifford-Klein forms of homogeneous spaces of $\mathrm{SO}(2, n)$, Internat. Math. Res. Notices 5 (2000) 235-251.

[OW] M.Osborne, G.Warner, The theory of Eisenstein systems, Pure and Applied Mathematics, 99. Academic Press, Inc. 1981, xiii+385 pp.

[Pan] P.Pansu, Métriques de Carnot-Carathéodory et quasiisométries des espaces symétriques de rang un, Ann. of Math. 129 (1989) 1-60.

[Pa] W.Parry, On some aspects of the theory of Anosov systems, Bull. Amer. Math. Soc. 42 (2005) 257-261.

[Pe] R.Penrose, Pentaplexity, Mathematical Intelligencer 2 (1979) 32-37.

[PS1] I.Piatetski-Shapiro, Discrete subgroups of Lie groups, Trans. Moscow Math. Soc. 18 (1968) 1-18.

[PS2] I.Piatetski-Shapiro, Automorphic functions and arithmetic groups, (Russian) Proc. Internat. Congr. Math. (Moscow, 1966) pp. 232-247. English translation, Amer. Math. Soc. Transl. 70 (1968) 185-201.

[PIR] V.Platonov, A.Rapinchuk, Algebraic groups and number theory, Pure and Applied Mathematics, 139. Academic Press, 1994. xii+614 pp.

[Pol] L.Polterovich, Growth of maps, distortion in groups and symplectic geometry, Invent. Math. 150 (2002) 655-686.

[Pop] S.Popa, Deformation and rigidity for group actions and von Neumann algebras, Proc. of ICM, vol. 1, 2006, pp. 445-477.

[Pr1] G.Prasad, Strong rigidity of Q-rank 1 lattices, Invent. Math. 21 (1973)255-286.

[Pr2] G.Prasad, Strong approximation for semi-simple groups over function fields, Ann. of Math. 105 (1977) 553-572.

[Rag1] M.S.Raghunathan, Discrete subgroups of Lie groups, Springer-Verlag, 1972. ix+227 pp.

[Rag2] M.S.Raghunathan, Discrete groups and Q-structures on semi-simple Lie groups, in Discrete subgroups of Lie groups and applications to moduli, pp. 225-321. Oxford Univ. Press, 1975.

[RaSS] A.Rapinchuk, Y.Segev, G.Seitz, Finite quotients of the multiplicative group of a finite dimensional division algebra are solvable, J. Amer. Math. Soc. 15 (2002) 929-978.

[Ra1] M.Ratner, Interactions between ergodic theory, Lie groups, and number theory, Proc, of International Congress of Mathematicians, Vol. 1, 2 (Zürich, 1994), pp. 157-182, Birkhäuser, 1995.

[Ra2] M.Ratner, Raghunathan's conjectures for Cartesian products of real and p-adic Lie groups, Duke Math. J. 77 (1995) 275-382.

[Ra3] M.Ratner, Raghunathan's topological conjecture and distributions of unipotent flows, Duke Math. J. 63 (1991) 235-280.

[Ra4] M.Ratner, Strict measure rigidity for unipotent subgroups of solvable groups, Invent. Math. 101 (1990) 449-482. 
[Ra5] M.Ratner, On measure rigidity of unipotent subgroups of semisimple groups, Acta. Math. 165 (1990) 229-309.

[Ra6] M.Ratner, On Raghunathan's measure conjecture, Ann. of Math. 134 (1991) 545-607.

[Ro] J.Rosenblatt, Uniqueness of invariant means for measure-preserving transformations, Trans. Amer. Math. Soc. 265 (1981) 623-636.

[Sar1] P.Sarnak, On cusp forms, in The Selberg trace formula and related topics, pp. 393-407, Contemp. Math., 53, Amer. Math. Soc., 1986.

[Sar2] P.Sarnak, Some applications of modular forms, Cambridge Tracts in Mathematics, 99. Cambridge University Press, 1990. x+111 pp.

[Sch] W.Schmidt, Metrical theorems on fractional parts of sequences, Trans. Amer. Math. Soc. 110 (1964) 493-518.

[Seg] Y.Segev, Shadows of elements, solvability of finite quotients and the Margulis-Platonov conjecture, in Groups, combinatorics \& geometry, pp. 257-267, World Sci. Publ., 2003.

[Se1] A.Selberg, Recent developments in the theory of discontinuous groups of motions of symmetric spaces, Lecture Notes in Mathematics, Vol. 118, pp. 99-120, Springer, 1970.

[Se2] A.Selberg, Discontinuous groups and harmonic analysis, Proc. Internat. Congr. Mathematicians (Stockholm, 1962), pp. 177-189, Inst. Mittag-Leffler, 1963.

[Se3] A.Selberg, On discontinuous groups in higher-dimensional symmetric spaces, in Contributions to function theory (internat. Colloq. Function Theory, Bombay, 1960), pp. 147-164, Tata Institute of Fundamental Research, 1960.

[Sen] M.Senechal, The mysterious Mr. Ammann, Math. Intelligencer 26 (2004) 10-21.

[Ser] J.P.Serre, Trees, Corrected 2nd printing of the 1980 English translation, Springer-Verlag, 2003. $\mathrm{x}+142 \mathrm{pp}$.

[Sh] Y.Shalom, The algebraization of Kazhdan's property (T), Prof. of ICM, vol. 2, 2006, pp. $1281-1310$.

[Si] C.Siegel, Some remarks on discontinuous groups, Ann. of Math. 46 (1945)708-718.

[Sp] R.Spatzier, An invitation to rigidity theory, in Modern dynamical systems and applications, pp. 211-231, Cambridge Univ. Press, 2004.

[Spr1] V.Sprindzuk, Achievements and problems of the theory of Diophantine approximations, Uspekhi Mat. Nauk 35 (1980), no. 4(214), 3-68, 248.

[Spr2] V.Sprindzuk, Metric theory of Diophantine approximations, A Halsted Press Book, John Wiley \& Sons, 1979. xiii+156 pp.

[St] J.Stallings, On torsion-free groups with infinitely many ends, Ann. of Math. 88 (1968) 312-334.

[Sul1] D.Sullivan, For $n>3$ there is only one finitely additive rotationally invariant measure on the $n$-sphere defined on all Lebesgue measurable subsets, Bull. Amer. Math. Soc. 4 (1981) 121-123.

[Sul2] D.Sullivan, Disjoint spheres, approximation by imaginary quadratic numbers, and the logarithm law for geodesics, Acta Math. 149 (1982) 215-237.

[Ti1] J.Tits, The work of Gregori Aleksandrovitch Margulis, Proceedings of the International Congress of Mathematicians (Helsinki, 1978), pp. 57-63, Acad. Sci. Fennica, Helsinki, 1980.

[Ti2] J.Tits, Free subgroups in linear groups, J. Algebra 20 (1972) 250-270.

[Vas] L.Vaserstein, Groups having the property T, Functional Anal. Appl. 2 (1968) 174.

[Vat] V. Vatsal, Uniform distribution of Heegner points, Invent. Math. 148 (2002) 146.

[Ve] T.Venkataramana, On superrigidity and arithmeticity of lattices in semisimple groups over local fields of arbitrary characteristic, Invent. Math. 92 (1988) 255-306.

[Vo] S.Vodopyanov, $\mathcal{P}$-differentiability on Carnot groups in different topologies and related topics, in Proceedings on Analysis and Geometry, pp. 603-670, Izdat. Ross. Akad. Nauk Sib. Otd. Inst. Mat., Novosibirsk, 2000. 
[Wa] Y.Watatani, Property $T$ of Kazhdan implies property FA of Serre, Math. Japon. 27 (1982) 97-103.

[We] A.Weil, On discrete subgroups of Lie groups. II, Ann. of Math. 75 (1962) 578-602.

[Ye] S.K.Yeung, Integrality and arithmeticity of co-compact lattice corresponding to certain complex two-ball quotients of Picard number one, Asian J. Math. 8 (2004) 107-129.

[Zi1] R.Zimmer, Ergodic theory and semisimple groups, Birkhäuser, 1984. x+209 pp.

[Zi2] R.Zimmer, Actions of semisimple groups and discrete subgroups, Proc. of International Congress of Mathematicians, Vol. 1, 2 (Berkeley, Calif., 1986), pp. 1247-1258, Amer. Math. Soc., 1987.

Lizhen Ji

Dept of Math

University of Michigan

Ann Arbor, MI 48109 